Journal for ImmunoTherapy of Cancer

\title{
Defining unique clinical hallmarks for immune checkpoint inhibitor- based therapies
}

\author{
Olivier Michielin, ${ }^{1}$ Aly-Khan Lalani, ${ }^{2}$ Caroline Robert, ${ }^{3,4}$ Padmanee Sharma, ${ }^{5}$ \\ Solange Peters ${ }^{1}$
}

To cite: Michielin 0, Lalani A$\mathrm{K}$, Robert $\mathrm{C}$, et al. Defining unique clinical hallmarks for immune checkpoint inhibitorbased therapies. Journal for ImmunoTherapy of Cancer 2022;10:e003024. doi:10.1136/ jitc-2021-003024

Accepted 22 October 2021

\section{SLinked}

http://dx.doi.org/10.1136/ bmjqs-2022-014870

Check for updates

(C) Author(s) (or their employer(s)) 2022. Re-use permitted under CC BY-NC. No commercial re-use. See rights and permissions. Published by BMJ.

${ }^{1}$ Department of Oncology, Lausanne University Hospital, Lausanne, Switzerland

${ }^{2}$ Department of Oncology, Juravinski Cancer Centre, McMaster University, Hamilton, Ontario, Canada

${ }^{3}$ Department of Medicine, Gustave Roussy Cancer

Campus, Villejuif, France

${ }^{4}$ Paris-Saclay University, Orsay, France

${ }^{5}$ Departments of Genitourinary Medical Oncology and Immunology, UT MD Anderson Cancer Center, Houston, Texas, USA

Correspondence to

Dr Olivier Michielin;

Olivier.Michielin@chuv.ch

\section{ABSTRACT}

Introduction Immuno-oncology therapies, including immune checkpoint inhibitors (ICls), have transformed cancer care and have brought into question whether classic oncology efficacy assessments adequately describe the distinctive responses observed with these agents. With more ICl-based therapies being approved across multiple tumor types, it is essential to define unique clinical hallmarks of these agents and their associated assessments to better reflect the therapeutic impact for both patients and physicians. Long-term survival and objective responses, such as depth and durability of responses, treatment-free survival, efficacy in brain metastases, improved health-related quality of life, and unique safety profiles, are among the hallmarks that have emerged for ICl therapies. An established clinical hallmark is a sustained long-term survival, as evidenced by a delayed separation of Kaplan-Meier survival curves, and a plateau at $\sim 3$ years. Combination ICI therapies provide the opportunity to raise this plateau, thereby affording durable survival benefits to more patients. Deepening of responses over time is a unique clinical ICI hallmark, with patients responding long term and with more durable complete responses. Depth of response has demonstrated prognostic value for long-term survival in some cancers, and several ICl studies have shown sustained responses even after discontinuing ICl therapy, offering the potential for treatment-free intervals. Although clinical evidence supporting efficacy in brain metastases is limited, favorable ICI intracranial responses have been seen that are largely concordant with extracranial responses. While patient outcomes can be significantly improved with ICls, they are associated with unique immune-mediated adverse reactions (IMARs), including delayed ICI toxicities, and may require multidisciplinary management for optimal care. Interestingly, patients discontinuing ICls for IMARs may maintain responses similar to patients who did not discontinue for an IMAR, whether they restarted ICI therapy or not.

Conclusion Herein, we comprehensively review and refine the clinical hallmarks uniquely associated with $\mathrm{ICl}$ therapies, which not only will rejuvenate our assessment of ICI therapeutic outcomes but also will lead to a greater appreciation of the effectiveness of $\mathrm{ICl}$ therapies.

\section{INTRODUCTION}

Immuno-oncology (I-O) therapy has revolutionized cancer treatment and has led to clinical responses unique to this class of agents. Contemporary I-O treatments include a range of therapies, such as immune checkpoint inhibitors (ICIs) and chimeric antigen receptor T-cell immunotherapies. ${ }^{1}$ With the discovery of checkpoint receptors involved in immune response regulation, ${ }^{2}$ ICIs have changed the landscape of immune activation and cancer treatment. Single-agent ICIs, including inhibitors of cytotoxic T-lymphocyte antigen 4 (CTLA-4), programmed death-1 (PD-1), and programmed death ligand 1 (PDL1), have demonstrated significant antitumor activity and are being combined with other agents to increase clinical benefit. ${ }^{34}$

With the first ICI clinical trials of the CTLA-4 inhibitor ipilimumab in melanoma, ${ }^{5}$ clinical hallmarks have emerged that are unique to ICI therapies (online supplemental graphical abstract, figure 1). Understanding clinical benefits associated with ICI now goes beyond traditional outcomes of survival and objective responses, though overall survival (OS) remains the gold standard endpoint. ${ }^{6}$ Other long-term measures, such as depth of response (DepOR), ${ }^{7}$ treatment-free survival (TFS), ${ }^{8} 9$ efficacy in brain metastases, ${ }^{10-15}$ improved health-related quality of life (HRQOL), ${ }^{16}$ and durable responses, have evolved as additional hallmarks associated with ICI-based therapies, with the possibility for a cure in some patients. ${ }^{5}$

ICI combinations bring the prospect of improved outcomes to specific tumor types and disease settings. Rationales for multimodal approaches can be conceptualized based on the cancer immunity cycle that highlights both the stepwise series of events that contribute to anticancer effects and opportunities to optimize clinical responses (figure 2). ${ }^{1718}$ However, responses to therapy across solid tumors are not uniform, ${ }^{18}$ and using a combination approach targeting multiple steps in the cycle may circumvent suboptimal responses. This emphasizes the rationale for combination therapies to 


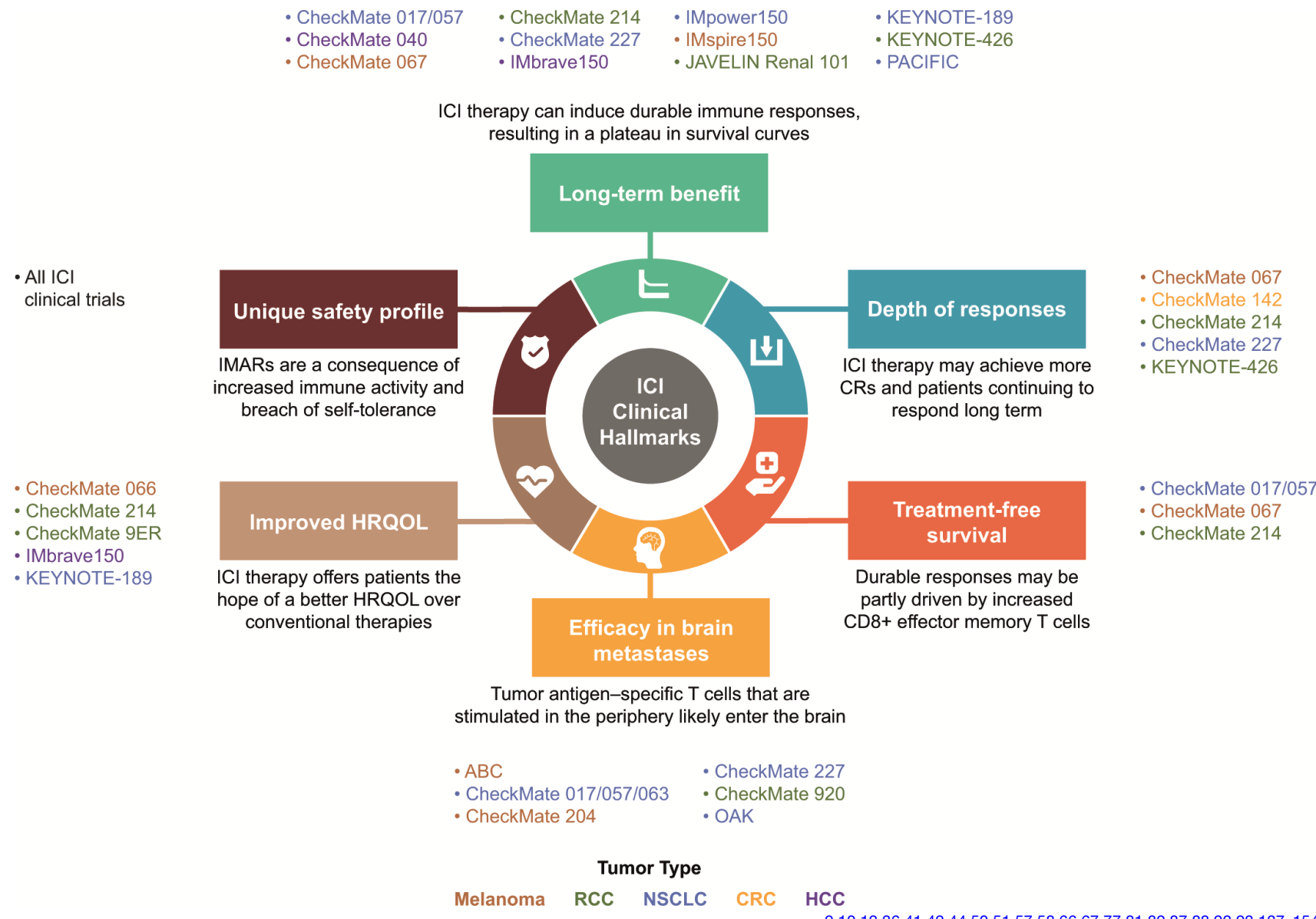

Figure 1 Clinical hallmarks of ICl-based therapies and representative studies.9 $10123641424450515758666777818287889293137-154$ CR, complete response; CRC, colorectal cancer; HCC, hepatocellular carcinoma; HRQOL, health-related quality of life; ICI, immune checkpoint inhibitor; IMAR, immune-mediated adverse reaction; NSCLC, non-small cell lung cancer; RCC, renal cell carcinoma.

address resistance mechanisms, the potential to target less immunogenic tumors (changing tumors from 'cold' (T-cell absence) to 'hot' (T-cell infiltration)), and to achieve greater clinical benefit than ICI monotherapy. ${ }^{19}$

In 2019, $76 \%$ of active PD-(L) 1 trials were testing combination regimens, including combination with other ICI therapies, targeted therapies, chemotherapies, and radiotherapy. ${ }^{20-24}$ Several ICIs are approved in combination with chemotherapies, including nivolumab plus ipilimumab for metastatic non-small cell lung cancer (NSCLC) in the USA and the European Union (EU), nivolumab for gastric and gastroesophageal junction cancers and esophageal adenocarcinoma in the USA, atezolizumab for metastatic non-squamous NSCLC, triple-negative breast cancer (TNBC), and small cell lung cancer in the USA and EU, and pembrolizumab for NSCLC, head and neck squamous cell carcinoma (HNSCC), esophageal and gastroesophageal junction cancer, and TNBC in the USA as well as NSCLC and HNSCC in the EU. ${ }^{21-23}$ 25-27 ICI combinations with targeted agents-for example, tyrosine kinase inhibitors (TKIs) or vascular endothelial growth factor (VEGF) inhibitors-have demonstrated impressive outcomes, resulting in Food and Drug Administration (FDA) approvals for advanced renal cell carcinoma (RCC) and HCG. ${ }^{21} 232428$ The rationale for combining anti-PD-1 and anti-CTLA-4 therapies is strong, as both antibodies employ distinct but complementary mechanisms to activate T-cell function and induce an antitumor effect. ${ }^{22} 2930$ The combination of nivolumab and ipilimumab is now FDA-approved for the treatment of melanoma, RCC, HCC, NSCLC, mesothelioma, and microsatellite instability-high/mismatch repair-deficient colorectal cancer (CRC). ${ }^{22}$ To improve clinical benefits for more patients, rational combination therapies will need to be designed based on an understanding of the mechanisms of action for each agent and its impact on the cancer immunity cycle. ${ }^{31}$

To comprehensively assess the effectiveness and outcomes of ICI therapies, we sought to refine the clinical hallmarks attributed to the ICI component of combination strategies (figure 1). There, indeed, is a need for increased awareness and broader inclusion of these hallmarks in clinical trial design. Investigation of measures inclusive of and beyond long-term survival, such as DepOR, TFS, efficacy in brain metastases, improved HRQOL, and a unique safety profile, have evolved as additional hallmarks of ICI-based therapies to take into consideration. 


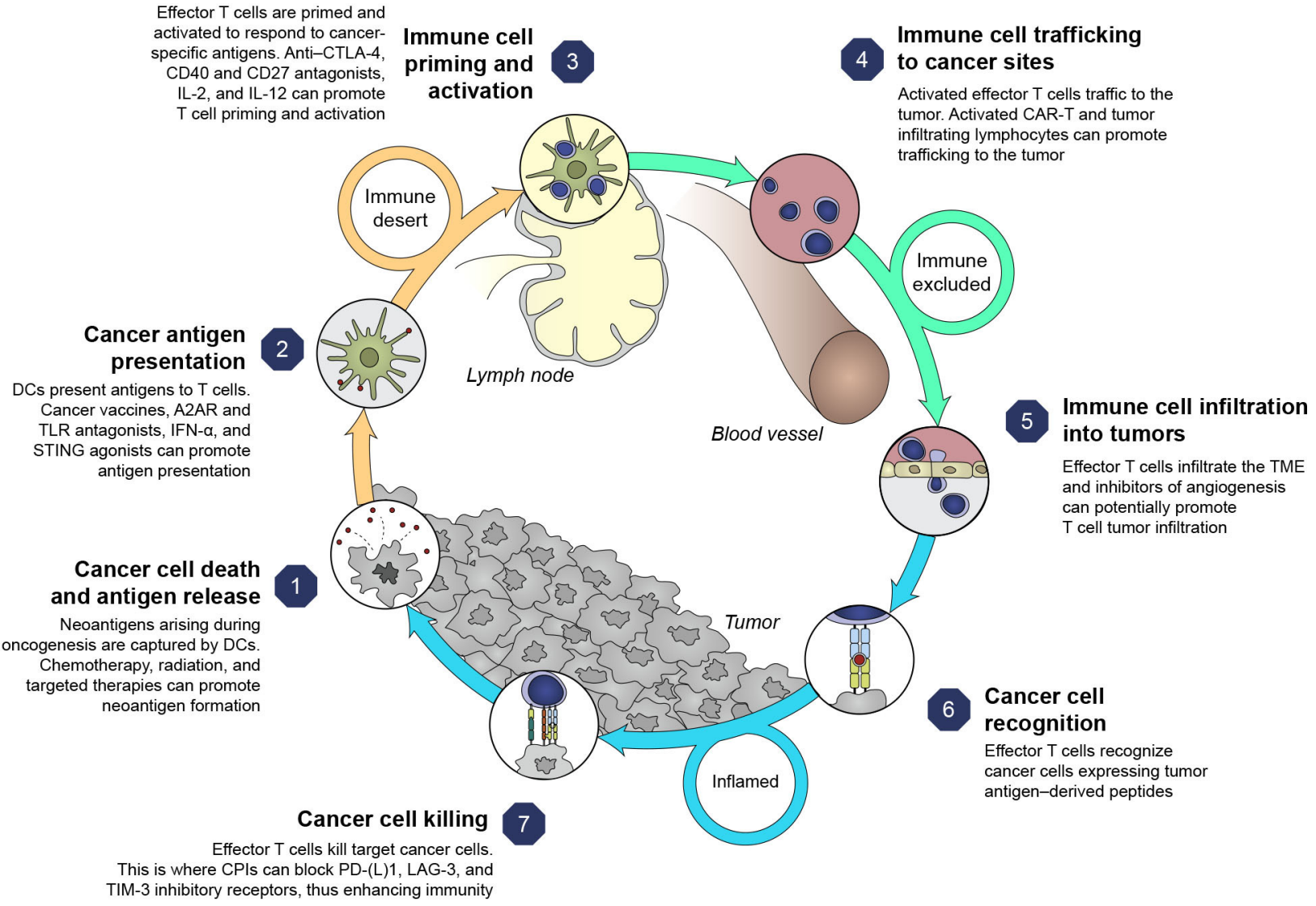

Figure 2 Cancer immunity cycle and therapy targets. ${ }^{17}$ Adapted from Chen and Mellman, ${ }^{17}$ with permission from Elsevier. A2AR, adenosine A2A receptor; CAR-T, chimeric antigen receptor T cell therapy; CD, cluster of differentiation; CTLA-4, cytotoxic T-lymphocyte antigen 4; DC, dendritic cell; ICI, immune checkpoint inhibitor; IFN- $\alpha$, interferon alpha; IL, interleukin; LAG-3, lymphocyte-activation gene three protein; PD-1, programmed death-1; PD-L1, programmed death ligand 1; STING, stimulator of interferon genes; TIM-3, T-cell immunoglobulin domain and mucin domain-3; TLR, toll-like receptor; TME, tumor microenvironment.

\section{CLINICAL HALLMARKS OF ICI THERAPIES Long-term survival benefits}

Compared with standard cytotoxic chemotherapy, ICIs restore active T-cell infiltration, increase T-cell repertoire diversification, and stimulate cancer-specific immune responses. ${ }^{32}{ }^{33}$ New benchmarks have been established with improved long-term survival and durable responses in significant proportions of patients, ${ }^{34-37}$ with the potential for TFS.

Survival benefits can be challenging to quantify, especially in trials with initial reporting based on short follow-up. However, with longer follow-up, an emerging clinical hallmark of ICI-based therapies is a delayed separation of Kaplan-Meier survival curves with an observed plateau beginning at $\sim 3$ years, demonstrating long-term survival benefits. ${ }^{5}$ This contrasts with chemotherapy, where early clinical responses are often seen but are short-lived. ${ }^{38}$ The first long-term ICI outcomes were reported for ipilimumab in advanced melanoma; despite low objective response rates (ORR), responses were durable, as shown by a plateau in the OS curve at $20 \%$ around 3 years, and the plateau was sustained at a 10-year follow-up. ${ }^{5}$ This brings the promise of a potential 'functional cure' in some patients with ongoing, long-term responses, even after treatment discontinuation. ${ }^{39}$ Several early ICI trials are now reporting data for up to 5 years or more, with similar plateaus seen at year $3 .^{36-44}$ Combination ICI therapies provide the opportunity to raise the plateau, thereby affording more patients durable OS benefits (figure 3 ).

Nivolumab plus ipilimumab has demonstrated improved survival and/or durable responses across multiple advanced tumors, including melanoma, ${ }^{36} \mathrm{HCC}^{45}$ DNA microsatellite instability-high/mismatch repair-deficient CRC, ${ }^{46}$ RCG, ${ }^{42}{ }^{43}$ and NSCLC. ${ }^{414}$ For example, landmark survival rates at 5 years showed that $52 \%$ of patients with advanced melanoma treated with nivolumab plus ipilimumab were alive, vs $44 \%$ and $26 \%$ receiving nivolumab or ipilimumab monotherapy, respectively. ${ }^{36}$ With a median follow-up of 55 months, superior OS, progression-free survival (PFS), and ORR benefits were maintained with nivolumab plus ipilimumab versus sunitinib for advanced RCC. ${ }^{43}$ The probability of response lasting $\geq 4$ years was higher with nivolumab plus ipilimumab versus sunitinib (59\% vs 30\%, respectively). ${ }^{43}$ Median OS with nivolumab plus ipilimumab was not reached (NR) vs 38.4 months with sunitinib, and 4-year probabilities were $53.4 \%$ vs $43.3 \%$, respectively. ${ }^{43}$ Nivolumab plus ipilimumab also 


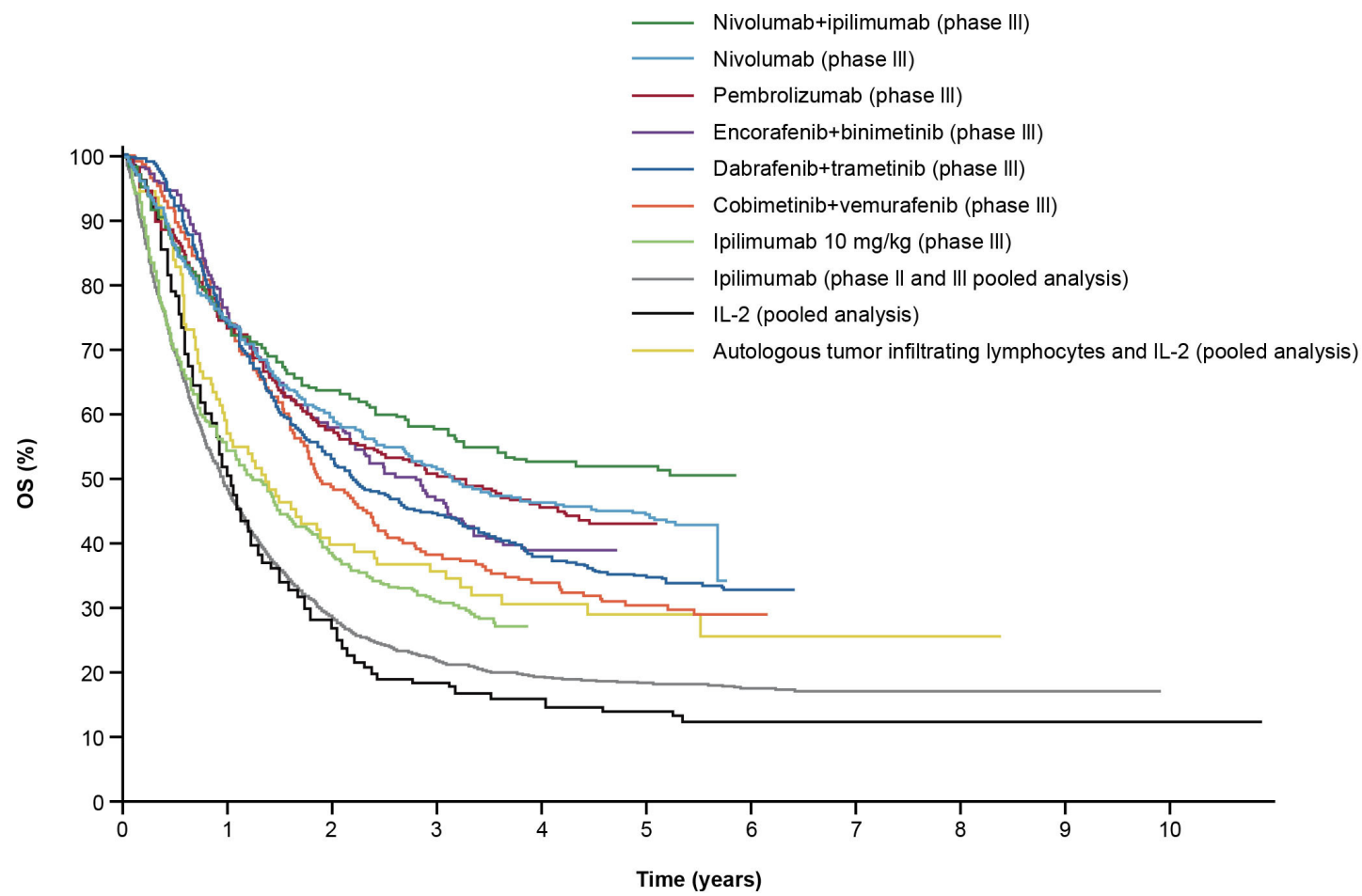

Figure 3 Long-term OS in clinical trials with immune checkpoint inhibitor and targeted therapies in patients with advanced melanoma. ${ }^{39}$ Data presented represent first-line treatment options, with the exception of those for IL-2 and pooled ipilimumab, for patients with melanoma; results will vary by tumor type. Permissions to use figures: licensed under the Creative Commons Attribution V.4.0 License (CC BY-NC 4.0) (https://creativecommons.org/licenses/by/4.0/). IL, interleukin; OS, overall survival.

demonstrated delayed PFS benefit, with curve separation favoring nivolumab plus ipilimumab in the tails of the curves; a plateau emerged at $\sim 30$ months with nivolumab plus ipilimumab, with the tails showing $\geq 10 \%$ separation between treatments. ${ }^{42}{ }^{43}$ Pembrolizumab plus ipilimumab demonstrated favorable survival in patients with melanoma at a 3-year follow-up, where 36-month PFS and OS were $59 \%$ and $73 \%$, respectively. ${ }^{48}$ Similarly, the combination of pembrolizumab plus axitinib in patients with RCC demonstrated significant OS (median NR vs 35.7 months, respectively; $\mathrm{p}=0.0003$ ) and PFS (median 15.4 vs 11.1 months, respectively; $\mathrm{p}<0.0001$ ) advantages over sunitinib. ${ }^{49}$ The OS curves for combination therapy separated early, and the risk of death was $\sim 32 \%$ lower with pembrolizumab plus axitinib versus sunitinib at a median follow-up of 30.6 months. ${ }^{49}$

After a minimum follow-up of 4 years in patients with NSCLC and PD-L1 $\geq 1 \%$, the median OS was longer with nivolumab plus ipilimumab (17.1 months) versus nivolumab (15.7 months) or chemotherapy (14.9 months). ${ }^{47}$ Similar results were seen in patients with a PD-L1 < $1 \%$ : median OS was 17.2 months with nivolumab plus ipilimumab vs 12.2 months with chemotherapy. ${ }^{41}$ After a minimum follow-up of 32.4 months, the median OS in patients with NSCLC was longer with atezolizumab plus bevacizumab plus chemotherapy versus bevacizumab plus chemotherapy: 19.8 vs 15 months, respectively. ${ }^{50}$ Pembrolizumab showed survival improvements with chemotherapy for advanced NSCLC. ${ }^{52}$ Pembrolizumab plus chemotherapy demonstrated favorable OS after a median follow-up of $\sim 4$ years: median OS was 22 months vs 10.6 months with chemotherapy. ${ }^{52}$ While cross-trial comparisons should not be made, many ICI combination therapies show improved efficacy outcomes versus monotherapies. Multimodal approaches that combine ICIs with agents such as chemotherapy, radiotherapy, TKIs, and ICIs have provided significant improvements in patient outcomes in some cases. Increasing baseline tumor burden was associated with significantly shorter OS and PFS. ${ }^{53}$ This supports the combined use of ICI therapies with debulking strategies, such as chemotherapy and radiotherapy. Combining two ICI therapies with differing modes of action may also allow for improved responses. For example, the roles of PD-1 and CTLA-4 in inhibiting immune responses, including antitumor responses, are largely distinct. CTLA-4 likely regulates T-cell proliferation early in an immune response, primarily in lymph nodes, whereas PD-1 suppresses T cells later in an immune response, primarily in peripheral tissues. ${ }^{54}$

\section{Increased DepOR over time}

DepOR is defined as the maximum percent tumor reduction from baseline and may prove useful as a measure of benefit that could provide an earlier read-out than timeto-event endpoints, providing an additional method of comparing treatment responses. ${ }^{55}$ Unlike conventional anticancer treatments, a hallmark of ICI-based therapies is a deepening of response over time, with more durable complete responses (CRs) and patients continuing to respond long-term. 


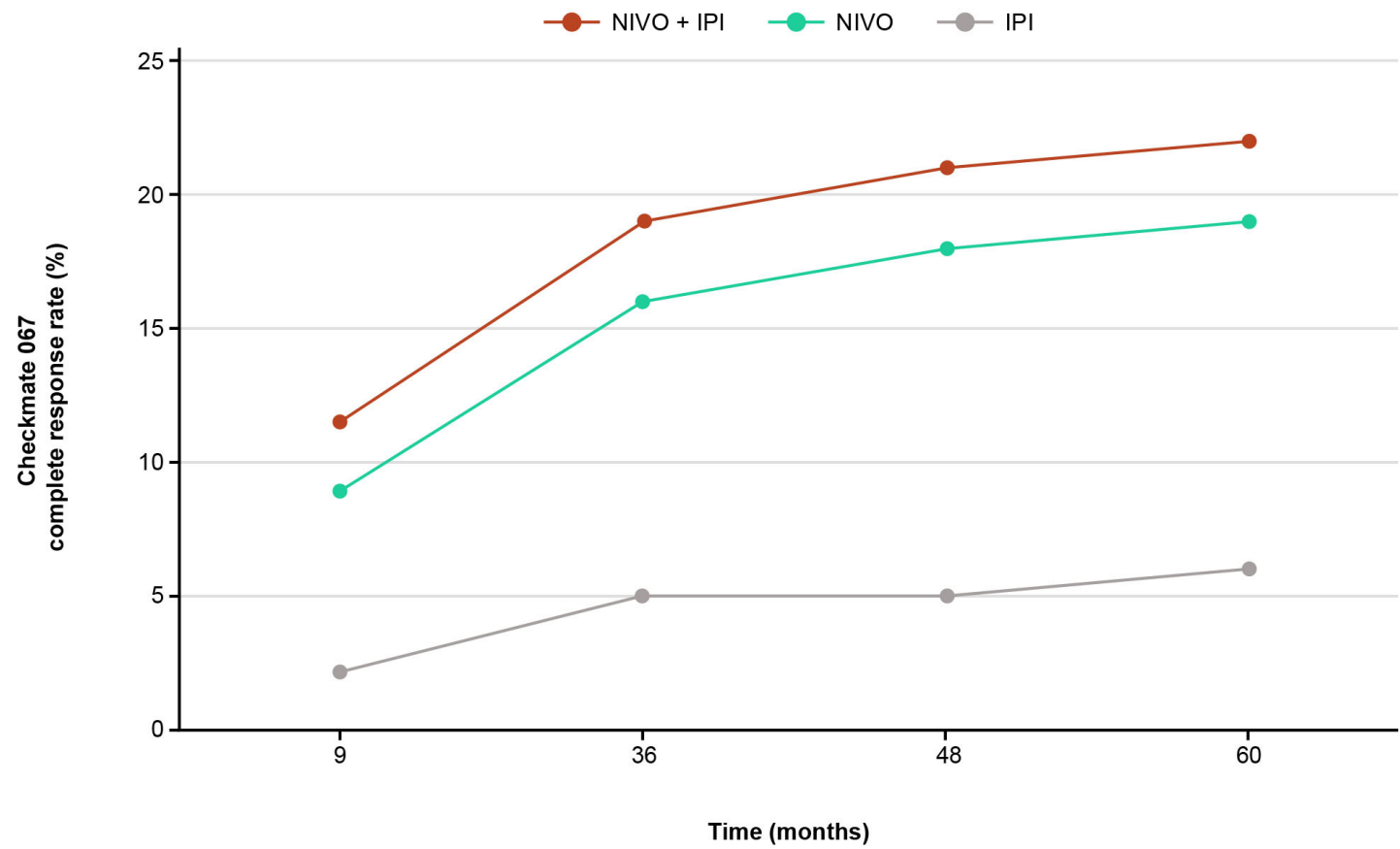

Figure 4 Patients with melanoma in CR over time. CR rates among patients undergoing immune checkpoint inhibitor therapy steadily increased, indicating that the best response can improve over time. ${ }^{36} 405758 \mathrm{CR}$, complete response; IPI, ipilimumab; NIVO, nivolumab.

DepOR has shown prognostic value for long-term survival in some cancers. In an FDA analysis of DepOR and survival in patients with advanced melanoma, a larger DepOR with ICI therapy correlated with a longer and higher rate of $\mathrm{OS}^{7}$ Another analysis of DepOR in patients with advanced NSCLC treated with a PD-1 inhibitor found that patients with higher percentages of maximal tumor shrinkage achieved higher OS benefits. ${ }^{55}$ Similarly, a combination of nivolumab plus ipilimumab in patients with advanced RCC also demonstrated a correlation between DepOR and OS. ${ }^{56}$

In patients treated with ICI combinations versus monotherapies, the CR rates are greater in general. With longer follow-up, higher CR rates are noted (figure 4), as seen with nivolumab plus ipilimumab in patients with melanoma, ${ }^{36} 405758$ RCC, ${ }^{43} 59$ and CRC. ${ }^{46} 60$ In patients with advanced melanoma, after a minimum follow-up of 9 months, the CR rate was higher with nivolumab plus ipilimumab versus either monotherapy, as was the ORR. ${ }^{57}$ After a follow-up of 5 years, ORR with nivolumab plus ipilimumab had not changed, but the percentage of CRs was greater, indicating an evolution of response as more patients with a partial response (PR) had converted to a CR over time. ${ }^{36}{ }^{57}$ In a pooled post hoc analysis of 5 -year data for nivolumab plus ipilimumab versus nivolumab monotherapy for advanced melanoma, patients with a CR in either arm at 12 months had an $85 \%-86 \%$ chance of being alive at 5 years, even without subsequent systemic therapy. ${ }^{61}$ An increasing proportion of patients with CRs was also observed with pembrolizumab in combination with ipilimumab in patients with advanced melanoma. ${ }^{482}$ While the ORR was preserved from a median follow-up of 17 months to 36.8 months, the $\mathrm{CR}$ rate almost doubled. ${ }^{48} 62$ This conversion of PRs to CRs appears more pronounced in patients on dual ICIs, where this hallmark is possibly driven by activation of a broad repertoire of T-cell receptors able to recognize unique tumor antigens $^{63}$ and increased CD4+/CD8+ effector memory $\mathrm{T}$ cells, ${ }^{64}$ reflecting a larger activated/memory T-cell diversity after ICI therapy.

\section{Treatment-free survival}

Several ICI studies demonstrate durable responses even after discontinuing combination therapy, resulting in prolonged treatment-free intervals, which may lessen potential for new or further toxicities and may have a costsaving impact. ${ }^{42-4465-67}$ TFS is a novel endpoint that has been defined as the time from treatment discontinuation to subsequent systemic therapy (treatment-free interval) or death (TFS, figure 5A) ${ }^{89}$ Patients who discontinue ICI therapy may experience durable disease control without need for subsequent systemic therapy ${ }^{68}$ However, thus far, for combination ICI therapy, this has only been published with nivolumab plus ipilimumab data, but not for ICIs plus TKIs or ICIs plus chemotherapy.

A study of nivolumab plus ipilimumab versus either monotherapy in advanced melanoma defined TFS as the area between Kaplan-Meier curves. ${ }^{8}$ To use these endpoints, the curves were divided into three phases: time on protocol therapy, TFS, and survival after subsequent therapy initiation (figure 5A). Curves were further subdivided to summarize the survival experience, free of anticancer therapy, with integration of toxicity data to characterize the patient's well-being. ${ }^{89}$ As illustrated in figure 5, a greater proportion of patients who had received nivolumab plus ipilimumab versus nivolumab 
A

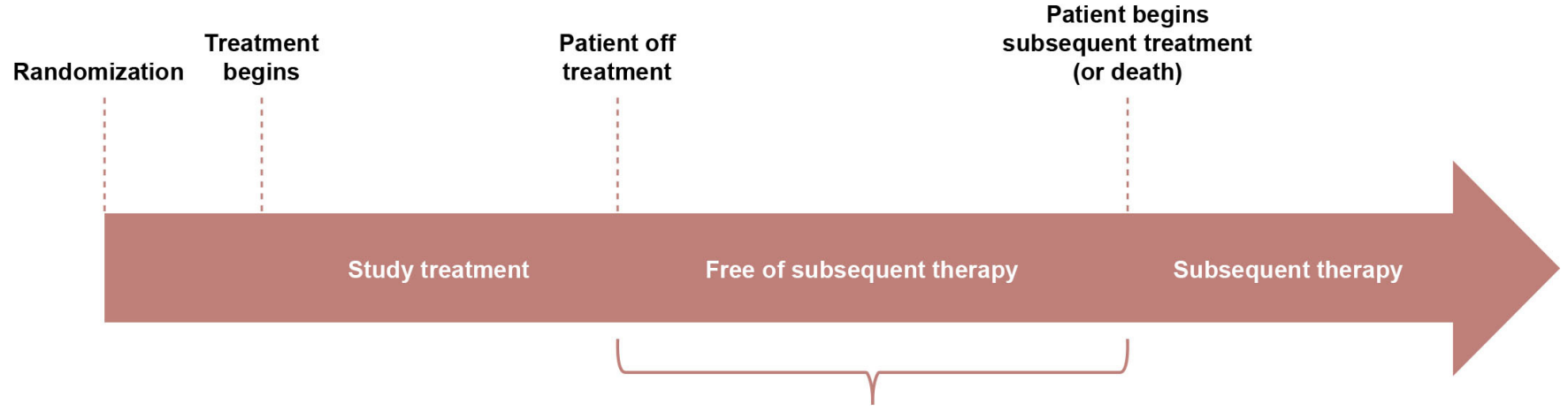

Treatment-free interval (survival)

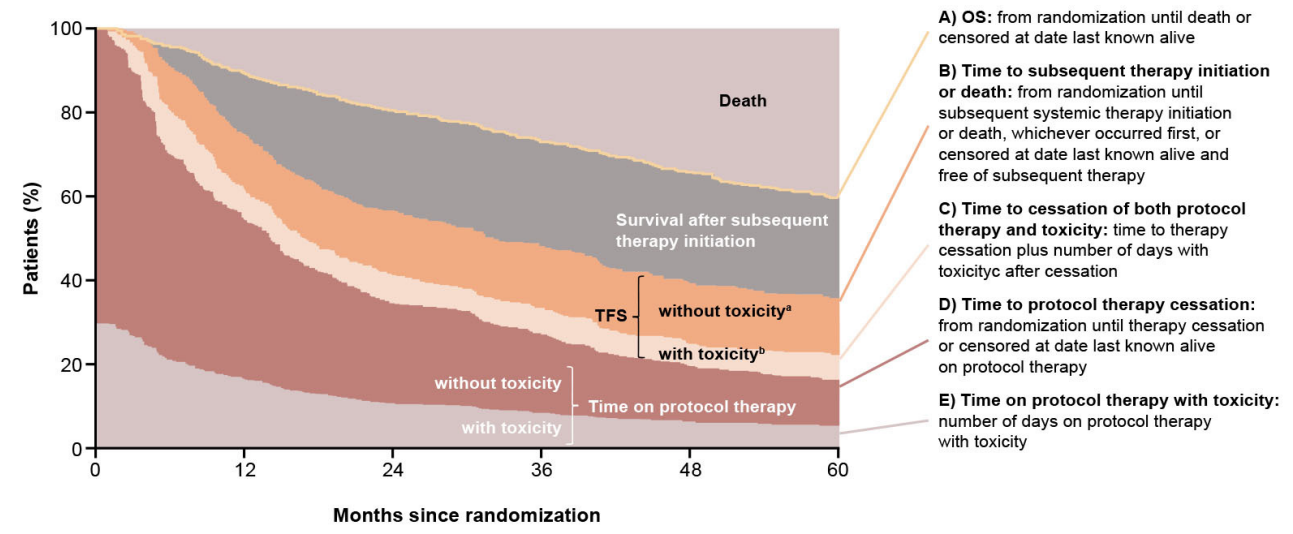

C

NIVO+IPI

(OS mean time, 38.6 months)

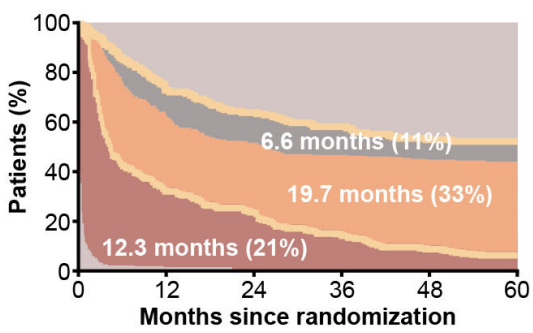

Time on protocol therapy
NIVO

(OS mean time, 36.1 months)

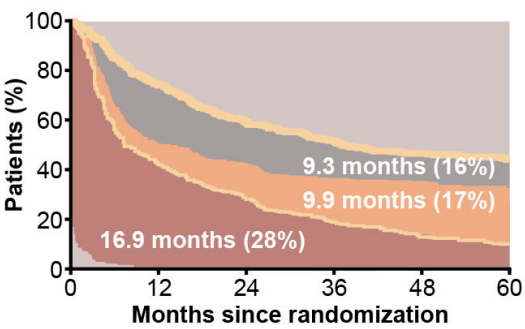

E

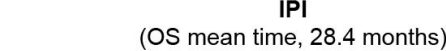

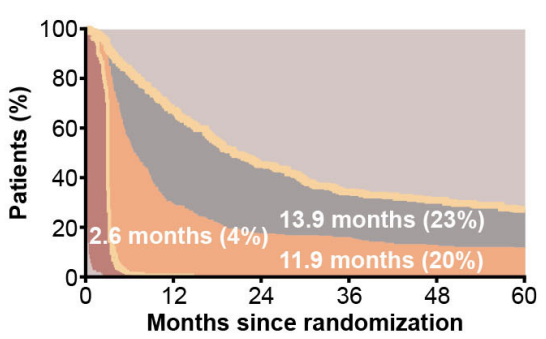

Treatment-free survival

Survival after subsequent therapy initiation

Figure 5 (A) Illustration of TFS assessment and OS curve partitioning. TFS estimates for (B) NIVO plus IPI, (C) nivolumab, and (D) ipilimumab in patients with melanoma in CheckMate 067 and 069. Data labels represent the mean number of months and the percentage of time in a treatment state during the 60 -month period. ${ }^{89}$ Areas are restricted mean times. ${ }^{\mathrm{a}}$ Time after cessation of protocol therapy without toxicity before initiation of subsequent systemic anticancer therapy or death. ${ }^{\mathrm{b}}$ Time after cessation of protocol therapy with toxicity while treatment-free. Permissions to use figures: licensed under the Creative Commons Attribution V.4.0 License (CC BY 4.0) (https://creativecommonsorg/licenses/by/40/). IPI, ipilimumab; NIVO, nivolumab; OS, overall survival; TFS, treatment-free survival.

or ipilimumab were alive and in TFS for up to 5 years (33\%, $17 \%$, and $20 \%$, respectively). ${ }^{9}$ Furthermore, the restricted mean TFS was longer for nivolumab plus ipilimumab versus either monotherapy (19.7, 9.9, and 11.9 months, respectively). ${ }^{8}$ Mean TFS with grade $\geq 3$ treatment-related adverse events (AEs) represented only a small proportion of the 60 -month period. ${ }^{9}$
In a 5-year analysis of patients with advanced melanoma, the treatment-free interval was 18.1 months with nivolumab plus ipilimumab but was only 1.8 months with nivolumab and 1.9 months with ipilimumab. ${ }^{36}$ Of patients alive at 5 years, $74 \%$ who received combination therapy were not receiving study drug or subsequent systemic therapy vs $58 \%$ with nivolumab and $45 \%$ with ipilimumab 
monotherapies. ${ }^{36}$ Among responders who discontinued study treatment for advanced RCC, a greater proportion who received nivolumab plus ipilimumab versus sunitinib did not require next-line therapy, including more with ongoing TFS. ${ }^{43} 67$ These results demonstrate the longterm TFS benefits of ICI therapy. Experience is currently limited to nivolumab plus ipilimumab and may be worthy of study with other combinations. Treatment-free intervals may factor in the shared decision making between patients and oncologists, as well as payers. Notably, the American Society of Clinical Oncology (ASCO) included treatment-free interval in the ASCO value framework, highlighting its importance as a key measure when assessing the value of cancer treatments. ${ }^{69}$

\section{Efficacy in brain metastases}

Brain metastases are a common complication of advanced malignancies that are difficult to treat and associated with poor outcomes. ${ }^{14}$ The role of ICIs in brain metastases is largely unknown, as these patients have traditionally been excluded from ICI clinical trials. The inflammatory tumor microenvironment (TME) of brain metastases is highly complex and immunosuppressive, and is comprised of interactions between diverse immune and cancer cells that may contribute to metastatic progression and impair therapeutic responses. ${ }^{70-74}$ Myeloid-derived suppressor cells (MDSCs), regulatory $\mathrm{T}$ cells (Tregs), and tumor-associated macrophages are recruited to the brain and play a negative role in antitumor immune responses. ${ }^{70} 71$ There can be PD-L1 expressed on cancer cells, T-cell activity downregulated due to tumor-induced T-cell exhaustion, and fewer tumor-infiltrating lymphocytes in brain metastases leading to the immunosuppressive environment. ${ }^{71-74}$ Historically, few chemotherapies and targeted therapies have shown efficacy in brain metastases. ${ }^{75}$ PD-(L) 1 therapies have been shown to reduce T-cell exhaustion in the brain. ${ }^{14}$ As intracranial tumor blood vessels are less permeable than extracranial vessels, ${ }^{76}$ ICI drugs likely cannot cross the blood-brain barrier; instead, tumor antigen-specific $\mathrm{T}$ cells are stimulated in the periphery and enter the brain subsequently. ${ }^{77}$

Clinical evidence supporting ICI efficacy in brain metastases is limited but has demonstrated favorable intracranial responses and survival, showing ICIs can have activity in brain metastases resulting from various solid tumors. ${ }^{10-15}$ Efficacy in brain metastases is being explored with pembrolizumab, nivolumab, atezolizumab, and durvalumab as monotherapies, which show promising activity. ${ }^{12} 13157879$ The first study of PD-1 inhibitors in untreated brain metastases was with pembrolizumab. Intracranial response rates were $22 \%$ and $33 \%$ for melanoma and NSCLC, respectively. ${ }^{15}$ Central nervous system responses were durable and were strongly concordant with systemic responses. ${ }^{15}$ Across initial singleagent ICI studies in patients with melanoma, NSCLC, or RCC, the median time from treatment onset to intracranial response was $\sim 2$ months. ${ }^{11}$ For patients with untreated brain metastases from RCC, intracranial response rates were $12 \%$ with nivolumab monotherapy; however, no objective response was reported in patients with brain lesions that were multiple or larger than $1 \mathrm{~cm} .{ }^{80}$

Combination therapies demonstrate increased intracranial efficacy versus ICI monotherapies. Intracranial ORR and CR seen with nivolumab plus ipilimumab in patients with melanoma were around double that with nivolumab monotherapy. ${ }^{10}$ Intracranial responses with nivolumab plus ipilimumab for asymptomatic, untreated melanoma brain metastases were largely concordant with extracranial responses and developed rapidly (median 2.3 months). ${ }^{11} 81$ The intracranial ORR was $57 \%$ (with $26 \%$ being CRs), and the overall ORR was 53\%. ${ }^{81}$ PFSs of 6 and 9 months were $64.2 \%$ and $59.5 \%$, respectively, for intracranial responses, and $75.9 \%$ and $70.4 \%$, respectively, for extracranial responses. ${ }^{81}$ OS was also maintained with ICI therapies in patients with brain metastases. For example, in patients with advanced NSCLC, survival and responses with nivolumab plus ipilimumab were comparable to patients without brain metastases. $^{82}$

Ongoing trials are investigating ICI therapy with radiotherapies for brain metastases, and evidence points to better outcomes with concurrent therapies. An analysis of patients undergoing stereotactic radiation therapy or stereotactic radiosurgery plus concurrent ICIs found a significant OS improvement with concurrent therapy versus non-concurrent therapy or radiotherapy alone, which may be associated with a decreased incidence of AEs and/or development of subsequent brain metastases. ${ }^{83} \mathrm{An}$ analysis of patients with brain metastases receiving stereotactic radiation therapy or stereotactic radiosurgery plus concurrent CTLA-4 or PD-(L) 1 inhibition reported significantly improved 1-year local control versus radiotherapy alone without increased risk of radiation necrosis. ${ }^{84}$ Time to new metastases was also significantly longer with concurrent PD-1 and/or CTLA-4 therapy and Gamma Knife radiosurgery. ${ }^{85}$ While radiotherapy plus ICIs may improve local brain metastasis control, more studies are needed to determine optimal timing and sequencing of radiotherapy and ICIs.

\section{Improved HRQOL}

ICI therapy favorably impacts HRQOL, which is important to evaluate the full cancer impact. In a systematic review of PD-(L) 1 therapies associated with consistent prolongation of time to symptomatic deterioration across therapies and solid tumors compared with cytotoxic therapy, PD-(L) 1 was associated with better symptomatic control at various follow-up points. ${ }^{16}$ With combination therapies showing greater toxicity, there is concern that HRQOL may be diminished. However, nivolumab plus ipilimumab maintained HRQOL in a manner similar to nivolumab monotherapy in patients with advanced melanoma at a 5-year analysis, despite having marked differences in rates of immune-mediated adverse reactions (IMARs). ${ }^{36} 86$ There were no meaningful sustained HRQOL deteriorations during or after treatment with nivolumab plus ipilimumab or nivolumab monotherapy. ${ }^{36}$ Patients with advanced RCC receiving nivolumab plus cabozantinib reported better HRQOL than patients receiving sunitinib. ${ }^{87}$ HRQOL was improved with 
atezolizumab plus bevacizumab versus sorafenib for unresectable HCC, leading to a delay in time to deterioration of HRQOL, physical functioning, and role functioning. ${ }^{88}$ Median time to deterioration of disease-related symptoms also took longer with combination treatment, and a lower proportion of patients experienced clinically meaningful symptom deterioration. ${ }^{88}$ However, improved HRQOL was not seen with all ICI combinations; for example, pembrolizumab plus axitinib and sunitinib monotherapy demonstrated similar HRQOL benefits for advanced RCC. ${ }^{89}$ Though data are limited, ICI therapy offers patients the potential of a better HRQOL over conventional therapies, related to the long-term benefits as well as the favorable safety profiles of ICI therapies. Notably, the European Society for Medical Oncology (ESMO) and ASCO included improvement in HRQOL in the ESMO-Magnitude of Clinical Benefit Scale and ASCO value framework, respectively, highlighting its importance as a key measure when assessing the value of cancer treatments. ${ }^{690}$

\section{Unique safety profile}

While patient outcomes can be significantly improved with ICI therapies, they are also associated with the emergence of unique autoimmune-like toxicities known as IMARs. ${ }^{91}$ Mechanisms of IMARs differ from AEs of non-ICI agents and are a consequence of increased immune activity and possibly a breach of self-tolerance. ${ }^{92} 93$ The precise mechanisms of IMARs are not fully understood, ${ }^{92} 93$ but they are likely similar to those promoting antitumor responses. ${ }^{93}$ Studies have shown that ICIs can induce T-cell diversification, expansion, and infiltration, affect B-cell responses and induction of autoantibodies, and promote cytokine responses, all of which may be involved in the pathophysiology of IMARs, though to what extent is unknown and this may differ between toxicities. ${ }^{92-97}$

ICI monotherapy is associated with significantly lower risk of overall and chemotherapy-related AEs (eg, fatigue, nausea, diarrhea, and hematological toxicities) and is overall better tolerated versus chemotherapy, with a significantly lower risk of any all-grade and grade 3-4 AEs; however, it is also associated with an increase in IMARs, including rash, pruritus, colitis, hypothyroidism, hyperthyroidism, and pneumonitis. ${ }^{98}$ While the presence of specific IMARs varies based on malignancy and inhibitor used, ${ }^{91}$ the most commonly affected organs are skin, gastrointestinal, and endocrine ${ }^{99}$; however, any organ may be affected, multiple IMARs may develop, and severe or life-threatening IMARs can occur. ${ }^{65} 9100$ Most IMARs manifest within the first 6 months of ICI therapy initiation ${ }^{65} 100$; however, late-onset IMARs may occur, even months after ICI discontinuation, and can render emerging diagnostics more complex. ${ }^{101}$ The term 'delayed immune-related events' was coined to characterize these late-onset events, defined as IMARs developing $\geq 90$ days after ICI discontinuation. ${ }^{101}$

While the safety profile of ICI monotherapies is well established, additional complexities arise with combination therapies where toxicities are increased, and it becomes challenging to identify which therapy underlies the toxicity. Combination CTLA-4/PD-(L) 1 results in a significantly higher frequency of IMARs with typically earlier onset versus monotherapies. ${ }^{65} 102103$ Each combination has a characteristic safety profile and usually results from a combination of the $\mathrm{AE}$ profiles of each monotherapy (figure 6). It

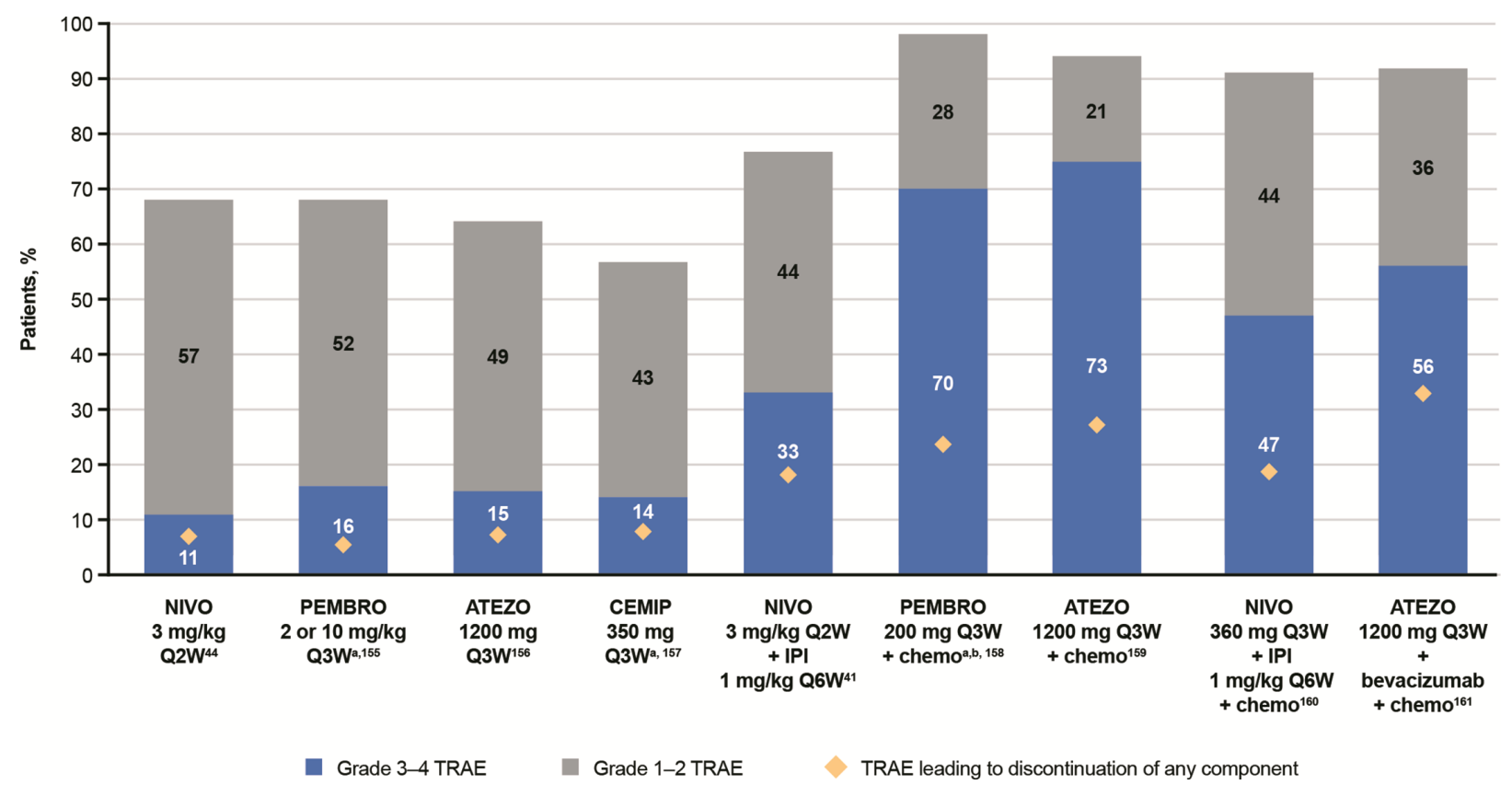

Figure 6 Safety profiles of ICI therapies in patients with non-small cell lung cancer (cross-trial comparisons cannot be made due to differences in dosing and methods used to assess TRAEs across studies). ${ }^{4144} 155-161$ a Data for grade 3-4 TRAEs also includes grade 5 TRAEs. ${ }^{b}$ Represents total adverse events, not just TRAEs. ATEZO, atezolizumab; CEMIP, cemiplimab; chemo, chemotherapy; ICI, immune checkpoint inhibitor; IPI, ipilimumab; NIVO, nivolumab; PEMBRO, pembrolizumab; QxW, every $\mathrm{x}$ weeks; TRAE, treatment-related adverse event. 
is important to properly assess the underlying cause of the toxicity, especially when therapies from different classes are used. For example, concomitant use of anti-PD-(L) 1 with a TKI requires an understanding of potential overlapping toxicities; while the management of TKI-related toxicity could be a dose interruption or reduction, IMARs are typically managed with corticosteroids. Anytime ICIs are given as backbones for combination treatments, IMARs can occur and should be treated as immune-mediated reactions. There is a need to identify biomarkers for patients who may or may not respond to ICI combinations to avoid the higher toxicities associated with combination therapies. Recently, realworld data have highlighted the importance of late-onset and long-lasting IMARs for patients receiving ICIs. ${ }^{104}$

\section{IMAR management guidelines}

IMAR management guidelines include those provided by the National Comprehensive Cancer Network, ${ }^{105}$ the Society for Immunotherapy of Cancer, ${ }^{106}$ and ESMO,${ }^{107}$ which provide treatment algorithms for common IMARs. Based on these guidelines, IMAR management requires close follow-up, patient engagement/self-management programs, and a multidisciplinary approach. Evidence-based IMAR management is limited and is an evolving area of research, with newer algorithms being proposed for severe and/or refractory cases. ${ }^{108}$ Using available management algorithms, we found that IMARs resolve in most patients, with the exception of endocrine IMARs. ${ }^{65}$ Common endocrine IMARs include thyroid dysfunction, hypophysitis, type 1 diabetes mellitus, and primary adrenal insufficiency, which, unlike non-endocrine IMARs, are often permanent and require lifelong hormone replacement. ${ }^{91}$

Notably, the presence of IMARs (skin and gastrointestinal toxicities in particular) has been associated with significantly improved survival and response outcomes with ICIs. ${ }^{94} 103109110$ However, results are conflicting, ${ }^{111} 112$ which may be related to a general lack of landmark analyses that can minimize the immortal time bias potentially associated with time-dependent factors, such as the development of IMARs. ${ }^{113} 114$

Studies have shown that patients who discontinue ICIs for IMARs maintain survival and response outcomes similar to patients who did not discontinue for an IMAR, whether they restarted ICI therapy or not. ${ }^{115116}$ Whether or not to re-treat patients following an IMAR is a frequent clinical scenario. Data are limited, but an analysis of patients with NSCLC who were re-treated with ICIs found that survival was significantly improved, and while $\sim 50 \%$ of patients developed a new or recurrent IMAR, they were mostly mild and manageable. ${ }^{116}$ The decision to re-treat patients is based on IMAR severity and sequelae, as supported by current guidelines, ${ }^{105-107}$ and should be made in consultation with patients and specialists.

\section{DISCUSSION}

ICI hallmarks, including long-term survival, deepening of responses over time, promising treatment-free intervals, brain metastases activity, and improved HRQOL, are emerging and enriching the metrics of benefits in many advanced cancers. With ICI therapies, more patients are responding and living longer, with the potential for more CRs, treatment-free intervals, and better HRQOL, hence the need for hallmarks of ICI combinations to be used more often in study designs and at the patient and upstream healthcare system levels. With more patients surviving long term, there is need for robust survivorship care plans. The long-term survival hallmark of ICI-based therapies is an observed plateau of Kaplan-Meier survival curves beginning at $\sim 3$ years, demonstrating longterm benefits. ${ }^{5}$ Until long-term survival data are available, additional hallmarks are important inputs into shared clinical decision making. DepOR has shown prognostic value for long-term survival, and a hallmark of ICI-based therapies is a deepening of response over time, with more CRs and patients continuing to respond long term. ${ }^{36} 485762$ Patients with advanced melanoma, CRC, or RCC receiving nivolumab plus ipilimumab demonstrated durable responses with longer treatment-free intervals, and a higher proportion of patients free of subsequent therapies were observed than patients receiving monotherapy. ${ }^{402} 43{ }^{45-67}$ Along with these beneficial hallmarks, immune system activation leads to a safety profile unique to ICIs. ${ }^{91}$ Notably, IMARs may be a biomarker for patient outcomes, as they are associated with survival and responses. ${ }^{94} 103109110$ IMARs also bring a need for multidisciplinary team awareness and recognition of ICI $\mathrm{AE}$ profiles and management for these patients.

Combination ICI therapies promise further survival advantages and aim to raise the plateau of the curve so that more patients experience sustained OS benefits. The combination treatment arena is advancing, including numerous new ICI therapies with multiple targets, including but not limited to VEGF/tyrosine kinase, ${ }^{66} 117$ the enzyme poly (ADP-ribose) polymerase, ${ }^{118}$ other checkpoints such as lymphocyteactivation gene 3 (LAG-3), ${ }^{119}$ T-cell immunoglobulin and mucin domain-3 (TIM-3), ${ }^{120}$ T-cell immunoreceptor with immunoglobulin and immunoreceptor tyrosine-based inhibitory motif (ITIM) domain (TIGIT), ${ }^{121}$ and other immune-modulating pathways (eg, IL-2 pathway agonist and toll-like receptors). More approvals are expected with ICI combinations across tumor types, and the clinical hallmarks will need to adapt to the changing field. This includes the potential for new or updated endpoints, including DepOR, TFS, and HRQOL, and in earlier disease, pathological CRs in the neoadjuvant setting and disease-free survival in the adjuvant setting. There is a need to understand the determinants driving response, resistance, and AEs, and to identify potential predictive biomarkers to optimize treatment.

ICI therapies display different response patterns and tumor response kinetics compared with cytotoxic and molecularly targeted agents, necessitating the use of alternative endpoints. In light of this, the FDA recognizes the use of traditional and surrogate endpoints to support accelerated approval of cancer therapies. ${ }^{122}$ In these settings, the FDA may grant approval based on a surrogate endpoint that is 'reasonably likely to predict clinical benefit'. ${ }^{\text {' } 22}$ In their 2018 guidance for industry, the FDA provides a robust discussion of oncology clinical trial endpoints and their limitations, 
including OS, PFS, ORR, CR, and those involving symptom assessment (HRQOL). ${ }^{122}$ The use of specific endpoints is guided by factors such as specific cancer, disease setting and location, size of effect and duration, DepOR, and available therapeutic options. ${ }^{122}$

As discussed by the FDA and other publications, there remain limitations with the clinical trial endpoints associated with the hallmarks of ICI therapy. For example, OS requires long follow-up periods in large trials and may be affected by crossover and subsequent therapies. ${ }^{122}$ With OS, there may also be a delay in benefit with ICI agents that may lead to a loss in statistical power, and conventional proportional hazards models may not appropriately capture ICI survival kinetics. ${ }^{6}$ Limitations associated with PFS include varying definitions among studies and being subject to assessment bias, measurement error, and missing data. ${ }^{6} 122$ PFS also requires frequent assessments and balanced timing of assessments among treatment arms and may not always correlate with survival. ${ }^{122}$ Similarly, definitions for ORR vary among studies, and ORR requires frequent assessments and balanced timing of assessments among treatment arms. ${ }^{122}$ ORR does not differentiate outcomes based on DepOR (CR vs PR), does not capture patients with durable stable disease, and may not always correlate with survival. ${ }^{6}{ }^{122}$ A limitation associated with DepOR is that its predictive value remains uncertain. ${ }^{123}$ Non-measurable lesions are not considered in the calculation of DepOR, which is based on measurement of tumor diameter. Additionally, reductions in target lesions do not always result in a diameter reduction, and tumor shrinkage may not always be symmetrical. ${ }^{123}$ For HRQOL, instruments currently used were designed for earlier chemotherapies and may not be sufficiently sensitive to capture important symptomatology unique to ICIs. ${ }^{122} 124$ Challenges with generalizing HRQOL data from prospective studies include limited data, heterogeneity in definitions and measurement tools, time point and duration of measurements, poor compliance, and missed data points between intervention and control arms. ${ }^{6} 122124$ Despite these limitations, the endpoints discussed for ICI trials are valuable for assessing the benefits of these therapies.

While there are encouraging responses in a subset of patients, many patients experience either primary refractory or acquired resistance after a response, in part because immune escape mechanisms can arise from defects in any or multiple steps of the cancer immunity cycle. ${ }^{18} 125126$ Resistance mechanisms are complex, involving tumor cellintrinsic and cell-extrinsic factors. Intrinsic factors include expression of tumor genes and pathways related to immune recognition, cell signaling, and expression of factors, preventing immune cell infiltration and/or function within the TME. ${ }^{127-129}$ Extrinsic mechanisms involve non-tumor components, including Tregs, MDSCs, macrophages, and inhibitory checkpoints. ${ }^{130-132}$ One goal of developing combination ICI therapies is to circumvent these resistance mechanisms and to achieve better outcomes for patients.

A major breakthrough with ICIs is the potential to achieve durable responses in some patients that can be maintained even years after ending treatment. A challenge with ICI response durability as an endpoint is that a unique definition is not available, and the optimal treatment duration in case of a durable response is unknown. Another challenge is whether ICI therapy should be given until disease progression or AEs, or whether treatment could be interrupted following a certain duration and/or achieving a radiological response. In metastatic melanoma, stopping ICI therapy before 2 years can be considered after $\geq 6$ months of treatment in patients with confirmed radiological control (stable disease or PR) in case of a complete pathological and/or metabolic response, according to the ESMO Guidelines Committee. ${ }^{133}$ Case reports of patients with advanced NSCLC who had an early response and received ICI therapy for $<2$ years reported ongoing benefits following discontinuation. ${ }^{134}$ A further challenge is that clinical trials using time-to-event endpoints are commonly designed with the assumption that the HRs between groups remain constant. The non-proportional hazards and delayed survival curve separation associated with ICI therapy reduces the ability for conventional statistical models to differentiate between them. ${ }^{135}$ Therefore, alternative methods are needed to shorten the study duration and/ or patient numbers needed to mitigate the effects. ${ }^{135}$ For survival curves with a plateau, cure models may be used to investigate heterogeneity between long-term survivors and patients not surviving long term. ${ }^{136}$

Notably, ASCO and ESMO included HRQOL in the ASCO value framework, and ESMO-Magnitude of Clinical Benefit Scale and ASCO also included TFS, emphasizing their importance as key measures when assessing the value of cancer treatments. ${ }^{6990}$ Evaluating the distinct hallmarks of ICI therapies and characterizing how they influence patients will inform future treatments. With ICI therapies being used in more patients and patients living longer, it is critical for regulatory bodies and treating physicians to recognize associated hallmarks to ensure appropriate outcomes and implement system-wide changes to address immune-mediated toxicities that require multidisciplinary management, including delayed ICI toxicities.

In conclusion, ICI-based therapies provide unique hallmarks that are associated with significantly improved clinical outcomes and a unique safety profile. Future research should focus on better exploiting these hallmarks with optimized dosing sequencing as we move into a treatment arena with new dual and triple combinations.

Correction notice This article has been corrected since it was first published online. Figures 1 and 6 have been updated. References 155 and 157 have been updated.

Acknowledgements The sponsor was involved in the review design and in the analysis and interpretation of data and information provided in the manuscript. However, the ultimate responsibility for opinions, data interpretation, and conclusions lies with the authors. Professional medical writing and editorial assistance were provided by Katherine Groschwitz, PhD, and Matthew Weddig of Spark Medica Inc, funded by Bristol Myers Squibb, according to Good Publication Practice 3 guidelines.

Contributors All authors were responsible for the opinions, data interpretation, and conclusions in this study.

Funding This work was supported by Bristol Myers Squibb. 
Competing interests SP received education grants and honoraria from and provided consultation, attended advisory boards, and/or provided lectures for AbbVie, Amgen, AstraZeneca, Bayer, Biocartis, Boehringer Ingelheim, Bristol Myers Squibb, Clovis, Daiichi Sankyo, Debiopharm, Eli Lilly, F. Hoffmann-La Roche, Foundation Medicine, Illumina, Incyte, Janssen, Merck Sharp and Dohme, Merck Serono, Merrimack, Novartis, Pharma Mar, Pfizer, Regeneron, Sanofi, Seattle Genetics, and Takeda. SP also talked at an organized public event for AstraZeneca, Boehringer Ingelheim, Bristol Myers Squibb, Eli Lilly, F. Hoffmann-La Roche, Illumina, Merck Sharp and Dohme, Novartis, Pfizer, Sanofi, and Takeda, and received grants and research supports as a (sub)investigator in trials (institutional financial support for clinical trials) sponsored by Amgen, AstraZeneca, Biodesix, Boehringer Ingelheim, Bristol Myers Squibb, Clovis, F. Hoffmann-La Roche, Illumina, Merck Sharp and Dohme, Merck Serono, Novartis, and Pfizer. A-KL received grants or research support, institutionally, from Bristol Myers Squibb, BioCanRx, Novartis, Roche, Ipsen, and EMD Serono, and was in an advisory or consulting role for AbbVie, Astellas, Bristol Myers Squibb, Eisai, Ipsen, Janssen, Merck, Novartis, Pfizer, Roche, and TerSera. OM received grants and personal fees from Bristol Myers Squibb and Merck Sharp and Dohme, Pierre-Fabre and Amgen, and personal fees from Roche, Novartis, GSK, and Merck, outside the submitted work. CR was a consultant for Amgen, Bristol Myers Squibb, Novartis, Roche, Merck Sharp and Dohme, Pierre Fabre, and AstraZeneca. PS has received consulting fees or stock ownership or attended advisory boards for Achelois, Adaptive Biotechnologies, Affini-T, Apricity, BioAtla, BioNTech, Codiak, Constellation, Dragonfly, Earli, Glympse, Hummingbird, ImaginAb, Infinity Pharma, Jounce, JSL Health, Lava Therapeutics, Lytix, Marker, Oncolytics, PBM Capital, Phenomics, Polaris, Sporos, Time BioVentures, and Venn Biosciences.

\section{Patient consent for publication Not applicable.}

Ethics approval This study does not involve human participants.

Provenance and peer review Commissioned; externally peer reviewed.

Supplemental material This content has been supplied by the author(s). It has not been vetted by BMJ Publishing Group Limited (BMJ) and may not have been peer-reviewed. Any opinions or recommendations discussed are solely those of the author(s) and are not endorsed by BMJ. BMJ disclaims all liability and responsibility arising from any reliance placed on the content. Where the content includes any translated material, BMJ does not warrant the accuracy and reliability of the translations (including but not limited to local regulations, clinical guidelines, terminology, drug names and drug dosages), and is not responsible for any error and/or omissions arising from translation and adaptation or otherwise.

Open access This is an open access article distributed in accordance with the Creative Commons Attribution Non Commercial (CC BY-NC 4.0) license, which permits others to distribute, remix, adapt, build upon this work non-commercially, and license their derivative works on different terms, provided the original work is properly cited, appropriate credit is given, any changes made indicated, and the use is non-commercial. See http://creativecommons.org/licenses/by-nc/4.0/.

\section{REFERENCES}

1 Pan C, Liu H, Robins E, et al. Next-generation immuno-oncology agents: current momentum shifts in cancer immunotherapy. J Hematol Oncol 2020;13:29.

2 Brunet JF, Denizot F, Luciani MF, et al. A new member of the immunoglobulin superfamily-CTLA-4. Nature 1987;328:267-70.

3 Kyi C, Postow MA. Immune checkpoint inhibitor combinations in solid tumors: opportunities and challenges. Immunotherapy 2016;8:821-37.

4 Ott PA, Hodi FS, Kaufman HL, et al. Combination immunotherapy: a road map. J Immunother Cancer 2017:5:16.

5 Schadendorf D, Hodi FS, Robert C, et al. Pooled analysis of long-term survival data from phase II and phase III trials of ipilimumab in unresectable or metastatic melanoma. J Clin Oncol 2015;33:1889-94.

6 Anagnostou V, Yarchoan M, Hansen AR, et al. Immuno-oncology trial endpoints: capturing clinically meaningful activity. Clin Cancer Res 2017;23:4959-69.

7 Osgood C, Mulkey F, Mishra-Kalyani PS, et al. FDA analysis of depth of response (DpR) and survival across 10 randomized controlled trials in patients with previously untreated unresectable or metastatic melanoma (UMM) by therapy type. J Clin Oncol 2019;37:9508.

8 Regan MM, Werner L, Rao S, et al. Treatment-free survival: a novel outcome measure of the effects of immune checkpoint inhibition-a pooled analysis of patients with advanced melanoma. J Clin Oncol 2019;37:3350-8.

9 Regan MM, Mantia C, Werner L, et al. Estimating treatmentfree survival (TFS) over extended follow-up in patients (pts) with advanced melanoma (MEL) treated with immune-checkpoint inhibitors (ICls): five-year follow-up of CheckMate 067. J Clin Oncol 2020;38:10043.

10 Long GV, Atkinson V, Lo S, et al. Combination nivolumab and ipilimumab or nivolumab alone in melanoma brain metastases: a multicentre randomised phase 2 study. Lancet Oncol 2018;19:672-81.

11 Harary M, Reardon DA, lorgulescu JB. Efficacy and safety of immune checkpoint blockade for brain metastases. CNS Oncol 2019;8:CNS33.

12 Gadgeel SM, Lukas RV, Goldschmidt J, et al. Atezolizumab in patients with advanced non-small cell lung cancer and history of asymptomatic, treated brain metastases: exploratory analyses of the phase III OAK study. Lung Cancer 2019;128:105-12.

13 Kluger HM, Chiang V, Mahajan A, et al. Long-term survival of patients with melanoma with active brain metastases treated with pembrolizumab on a phase II trial. J Clin Oncol 2019;37:52-60.

14 Aquilanti E, Brastianos PK. Immune checkpoint inhibitors for brain metastases: a primer for neurosurgeons. Neurosurgery 2020;87:e281-8.

15 Goldberg SB, Gettinger SN, Mahajan A, et al. Pembrolizumab for patients with melanoma or non-small-cell lung cancer and untreated brain metastases: early analysis of a non-randomised, open-label, phase 2 trial. Lancet Oncol 2016;17:976-83.

16 Abdel-Rahman O, Oweira H, Giryes A. Health-related quality of life in cancer patients treated with PD-(L)1 inhibitors: a systematic review. Expert Rev Anticancer Ther 2018;18:1231-9.

17 Chen DS, Mellman I. Oncology meets immunology: the cancerimmunity cycle. Immunity 2013;39:1-10.

18 Gao D. Compound-therapy based on cancer-immunity cycle: promising prospects for antitumor regimens. Am J Cancer Res 2019;9:212-8.

19 Gerard CL, Delyon J, Wicky A, et al. Turning tumors from cold to inflamed to improve immunotherapy response. Cancer Treat Rev 2021;101:102227.

20 Xin Yu J, Hodge JP, Oliva C, et al. Trends in clinical development for PD-1/PD-L1 inhibitors. Nat Rev Drug Discov 2020;19:163-4.

21 Genentech Inc. TECENTRIQ® (atezolizumab) [package insert], 2021https://www.gene.com/download/pdf/tecentriq_prescribing. pdf

22 Bristol Myers Squibb. OPDIVO® (nivolumab) [package insert], 2021 Available: https://packageinserts.bms.com/pi/pi_opdivo.pdf

23 Merck \& Co Inc. KEYTRUDA® (pembrolizumab) [package insert], 2021. Available: https://www.merck.com/product/usa/pi_circulars/ k/keytruda/keytruda_pi.pdf

24 EMD Serono Inc, Pfizer Inc. BAVENCIO® (avelumab) [package insert], 2020. Available: https://www.emdserono.com/us-en/pi/ bavencio-pi.pdf

25 Bristol Myers Squibb. Opdivo $®$ (nivolumab) summary of product characteristics 2020.

26 Genentech Inc. TECENTRIQ® (atezolizumab) [summary of product characteristics], 2020. Available: https://www.ema.europa.eu/ en/documents/product-information/tecentriq-epar-productinformation_en.pdf

27 Merck Sharp \& Dohme Limited. KEYTRUDA® (pembrolizumab) [summary of product characteristics], 2021. Available: https://www. ema.europa.eu/documents/product-information/keytruda-eparproduct-information_en.pdf

28 Motzer RJ, Robbins PB, Powles T, et al. Avelumab plus axitinib versus sunitinib in advanced renal cell carcinoma: biomarker analysis of the phase 3 javelin renal 101 trial. Nat Med 2020;26:1733-41

29 Hui E, Cheung J, Zhu J, et al. T cell costimulatory receptor CD28 is a primary target for PD-1-mediated inhibition. Science 2017;355:1428-33.

30 Yokosuka T, Kobayashi W, Takamatsu M, et al. Spatiotemporal basis of CTLA-4 costimulatory molecule-mediated negative regulation of T cell activation. Immunity 2010;33:326-39.

31 Sharma P, Siddiqui BA, Anandhan S, et al. The next decade of immune checkpoint therapy. Cancer Discov 2021;11:838-57.

32 Quezada SA, Peggs KS, Curran MA, et al. CTLA4 blockade and GM-CSF combination immunotherapy alters the intratumor balance of effector and regulatory T cells. J Clin Invest 2006;116:1935-45.

33 Li B, VanRoey M, Wang C, et al. Anti-programmed death-1 synergizes with granulocyte macrophage colony-stimulating factor-secreting tumor cell immunotherapy providing therapeutic benefit to mice with established tumors. Clin Cancer Res 2009;15:1623-34. 
34 Robert C, Ribas A, Schachter J, et al. Pembrolizumab versus ipilimumab in advanced melanoma (KEYNOTE-006): post-hoc 5 -year results from an open-label, multicentre, randomised, controlled, phase 3 study. Lancet Oncol 2019:20:1239-51.

35 Hamid O, Robert C, Daud A, et al. Five-year survival outcomes for patients with advanced melanoma treated with pembrolizumab in KEYNOTE-001. Ann Oncol 2019;30:582-8.

36 Larkin J, Chiarion-Sileni V, Gonzalez R, et al. Five-year survival with combined nivolumab and ipilimumab in advanced melanoma. N Engl J Med 2019;381:1535-46.

37 Topalian SL, Hodi FS, Brahmer JR, et al. Five-year survival and correlates among patients with advanced melanoma, renal cell carcinoma, or non-small cell lung cancer treated with nivolumab. JAMA Oncol 2019;5:1411-20.

38 Quinn C, Garrison LP, Pownell AK, et al. Current challenges for assessing the long-term clinical benefit of cancer immunotherapy: a multi-stakeholder perspective. J Immunother Cancer 2020;8:e000648.

39 Michielin O, Atkins MB, Koon HB, et al. Evolving impact of longterm survival results on metastatic melanoma treatment. $J$ Immunother Cancer 2020;8:e000948.

40 Hodi FS, Chiarion-Sileni V, Gonzalez R, et al. Nivolumab plus ipilimumab or nivolumab alone versus ipilimumab alone in advanced melanoma (CheckMate 067): 4-year outcomes of a multicentre, randomised, phase 3 trial. Lancet Oncol 2018;19:1480-92.

41 Hellmann MD, Paz-Ares L, Bernabe Caro R, et al. Nivolumab plus ipilimumab in advanced non-small-cell lung cancer. N Engl J Med 2019;381:2020-31.

42 Tannir NM, McDermott DF, Escudier B, et al. Overall survival and independent review of response in CheckMate 214 with 42-month follow-up: first-line nivolumab + ipilimumab $(\mathrm{N}+\mathrm{l})$ versus sunitinib $(\mathrm{S})$ in patients (pts) with advanced renal cell carcinoma (aRCC). J Clin Oncol 2020;38:609.

43 Albiges L, Tannir NM, Burotto M, et al. Nivolumab plus ipilimumab versus sunitinib for first-line treatment of advanced renal cell carcinoma: extended 4-year follow-up of the phase III CheckMate 214 trial. ESMO Open 2020;5:e001079.

44 Borghaei H, Gettinger S, Vokes EE, et al. Five-year outcomes from the randomized, phase III trials CheckMate 017 and 057: nivolumab versus docetaxel in previously treated non-small-cell lung cancer. $J$ Clin Oncol 2021;39:723-33.

45 Yau T, Kang Y-K, Kim T-Y. Nivolumab (NIVO) + ipilimumab (IPI) combination therapy in patients (pts) with advanced hepatocellular carcinoma (aHCC): results from CheckMate 040. J Clin Oncol 2020;37:4012.

46 Overman MJ, Lonardi S, Wong KYM, et al. Nivolumab (NIVO) + low-dose ipilimumab (IPI) in previously treated patients (PTS) with microsatellite instability-high/mismatch repair-deficient (MSI-H/ dMMR) metastatic colorectal cancer (mCRC): long-term follow-up. $J$ Clin Oncol 2019;37:635.

47 Paz-Ares LG, Ciuleanu T-E, Lee J-S. Nivolumab (NIVO) plus ipilimumab (IPI) versus chemotherapy (chemo) as first-line (1L) treatment for advanced non-small cell lung cancer (NSCLC): 4-year update from CheckMate 227. J Clin Oncol 2021;39:9016.

48 Carlino MS, Menzies AM, Atkinson V, et al. Long-term follow-up of standard-dose pembrolizumab plus reduced-dose ipilimumab in patients with advanced melanoma: KEYNOTE-029 part 1B. Clin Cancer Res 2020;26:5086-91.

49 Powles T, Plimack ER, Soulières D, et al. Pembrolizumab plus axitinib versus sunitinib monotherapy as first-line treatment of advanced renal cell carcinoma (KEYNOTE-426): extended followup from a randomised, open-label, phase 3 trial. Lancet Oncol 2020;21:1563-73.

50 Socinski MA, Mok TS, Nishio M. Abstract CT216: IMpower150 final analysis: efficacy of atezolizumab (atezo) + bevacizumab (bev) and chemotherapy in first-line (1L) metastatic nonsquamous (nsq) nonsmall cell lung cancer (NSCLC) across key subgroups. Cancer Res 2020;80:CT216.

51 Gandhi L, Rodríguez-Abreu D, Gadgeel S, et al. Pembrolizumab plus chemotherapy in metastatic non-small-cell lung cancer. N Engl $J$ Med 2018;378:2078-92.

52 Gray J, Rodríguez-Abreu D, Powell SF, et al. FP13.02 pembrolizumab + pemetrexed-platinum vs pemetrexed-platinum for metastatic NSCLC: 4-year follow-up from KEYNOTE-189. J Thorac Oncol 2021:16:S224.

53 Katsurada M, Nagano T, Tachihara M, et al. Baseline tumor size as a predictive and prognostic factor of immune checkpoint inhibitor therapy for non-small cell lung cancer. Anticancer Res 2019;39:815.

54 Horvath L, Thienpont B, Zhao L, et al. Overcoming immunotherapy resistance in non-small cell lung cancer (NSCLC) - novel approaches and future outlook. Mol Cancer 2020;19:141.
55 McCoach CE, Blumenthal GM, Zhang L, et al. Exploratory analysis of the association of depth of response and survival in patients with metastatic non-small-cell lung cancer treated with a targeted therapy or immunotherapy. Ann Oncol 2017;28:2707-14.

56 Grünwald V, Choueiri TK, Rini BI, et al. Association between depth of response and overall survival: exploratory analysis in patients with previously untreated advanced renal cell carcinoma (aRCC) in CheckMate 214. Ann Oncol 2019;30:v382-3.

57 Larkin J, Chiarion-Sileni V, Gonzalez R, et al. Combined nivolumab and ipilimumab or monotherapy in untreated melanoma. $N$ Engl $J$ Med 2015;373:23-34

58 Wolchok JD, Chiarion-Sileni V, Gonzalez R, et al. Overall survival with combined nivolumab and ipilimumab in advanced melanoma. N Engl J Med 2017;377:1345-56.

59 Motzer RJ, Tannir NM, McDermott DF, et al. Nivolumab plus ipilimumab versus sunitinib in advanced renal-cell carcinoma. N Engl J Med 2018;378:1277-90.

60 Overman MJ, Bergamo F, McDermott RS, et al. Nivolumab in patients with DNA mismatch repair-deficient/microsatellite instability-high (dMMR/MSI-H) metastatic colorectal cancer (mCRC): long-term survival according to prior line of treatment from CheckMate-142. J Clin Oncol 2018;36:554.

61 Robert C, Long GV, Larkin J. 1082MO 5-year characterization of complete responses in patients with advanced melanoma who received nivolumab plus ipilimumab (NIVO+IPI) or NIVO alone. Ann Oncol 2020;31:S734-5.

62 Long GV, Atkinson V, Cebon JS, et al. Standard-dose pembrolizumab in combination with reduced-dose ipilimumab for patients with advanced melanoma (KEYNOTE-029): an open-label, phase 1B trial. Lancet Oncol 2017;18:1202-10.

63 Robert L, Tsoi J, Wang X, et al. CTLA4 blockade broadens the peripheral T-cell receptor repertoire. Clin Cancer Res 2014;:20:2424-32.

64 Ohmura H, Yamaguchi K, Hanamura F. Activation of central/effector memory $T$ cells in advanced gastric cancer patients treated with antiprogrammed death-1 antibody. J Clin Oncol 2019;37:54

65 Overman MJ, Lonardi S, Wong KYM, et al. Durable clinical benefit with nivolumab plus ipilimumab in DNA mismatch repair-deficient/ microsatellite instability-high metastatic colorectal cancer. J Clin Oncol 2018;36:773-9.

66 Rini BI, Plimack ER, Stus V, et al. Pembrolizumab plus axitinib versus sunitinib for advanced renal-cell carcinoma. $N$ Engl J Med 2019;380:1116-27.

67 Regan M, Jegede OA, Mantia C, et al. 713P Treatment-free survival, with and without toxicity, after immuno-oncology vs targeted therapy for advanced renal cell carcinoma (aRCC): 42-month results of CheckMate 214. Ann Oncol 2020;31:S561.

68 Motzer RJ, Escudier B, McDermott DF, et al. Survival outcomes and independent response assessment with nivolumab plus ipilimumab versus sunitinib in patients with advanced renal cell carcinoma: 42-month follow-up of a randomized phase 3 clinical trial. $J$ Immunother Cancer 2020;8:e000891.

69 Schnipper LE, Davidson NE, Wollins DS, et al. Updating the American Society of Clinical Oncology Value Framework: revisions and reflections in response to comments received. $J$ Clin Oncol 2016;34:2925-34

70 Lindau D, Gielen P, Kroesen M, et al. The immunosuppressive tumour network: myeloid-derived suppressor cells, regulatory $T$ cells and natural killer T cells. Immunology 2013;138:105-15.

71 Hambardzumyan D, Gutmann DH, Kettenmann H. The role of microglia and macrophages in glioma maintenance and progression. Nat Neurosci 2016;19:20-7.

72 Ogiya R, Niikura N, Kumaki N, et al. Comparison of immune microenvironments between primary tumors and brain metastases in patients with breast cancer. Oncotarget 2017;8:103671-81.

73 Di Giacomo AM, Valente M, Cerase A, et al. Immunotherapy of brain metastases: breaking a "dogma". J Exp Clin Cancer Res 2019:38:419.

74 Farber SH, Tsvankin V, Narloch JL, et al. Embracing rejection: immunologic trends in brain metastasis. Oncoimmunology 2016;5:e1172153

75 Brastianos P, Davies MA, Margolin K, et al. Modern management of central nervous system metastases in the era of targeted therapy and immune oncology. Am Soc Clin Oncol Educ Book 2019;39:e59-69.

76 Lockman PR, Mittapalli RK, Taskar KS, et al. Heterogeneous blood-tumor barrier permeability determines drug efficacy in experimental brain metastases of breast cancer. Clin Cancer Res 2010;16:5664-78

77 Taggart D, Andreou T, Scott KJ, et al. Anti-PD-1/anti-CTLA-4 efficacy in melanoma brain metastases depends on extracrania 
disease and augmentation of $\mathrm{CD} 8^{+} \mathrm{T}$ cell trafficking. Proc Natl Acad Sci U S A 2018:115:E1540-9.

78 Wang S, Hu C, Xie F, et al. Use of programmed death receptor-1 and/or programmed death ligand 1 inhibitors for the treatment of brain metastasis of lung cancer. Onco Targets Ther 2020;13:667-83.

79 Crinò L, Bronte G, Bidoli P, et al. Nivolumab and brain metastases in patients with advanced non-squamous non-small cell lung cancer. Lung Cancer 2019;129:35-40.

80 Flippot R, Dalban C, Laguerre B, et al. Safety and efficacy of nivolumab in brain metastases from renal cell carcinoma: results of the GETUG-AFU 26 NIVOREN multicenter phase II study. J Clin Oncol 2019;37:2008-16.

81 Tawbi HA, Forsyth PA, Algazi A, et al. Combined nivolumab and ipilimumab in melanoma metastatic to the brain. $N$ Engl $\mathrm{J}$ Med 2018;379:722-30.

82 Borghaei H, Pluzanski A, Caro RB. Abstract CT221: nivolumab (NIVO) + ipilimumab (IPI) as first-line (1L) treatment for patients with advanced non-small cell lung cancer (NSCLC) with brain metastases: results from CheckMate 227. Cancer Res 2020;80:CT221.

83 Chen L, Douglass J, Kleinberg L, et al. Concurrent immune checkpoint inhibitors and stereotactic radiosurgery for brain metastases in non-small cell lung cancer, melanoma, and renal cell carcinoma. Int J Radiat Oncol Biol Phys 2018;100:916-25.

84 Kowalski ES, Remick JS, Sun K, et al. Immune checkpoint inhibition in patients treated with stereotactic radiation for brain metastases. Radiat Oncol 2020;15:245.

85 Gatterbauer B, Hirschmann D, Eberherr N, et al. Toxicity and efficacy of gamma knife radiosurgery for brain metastases in melanoma patients treated with immunotherapy or targeted therapy-A retrospective cohort study. Cancer Med 2020;9:4026-36.

86 Schadendorf D, Larkin J, Wolchok J, et al. Health-related quality of life results from the phase III CheckMate 067 study. Eur J Cancer 2017;82:80-91.

87 Choueiri TK, Powles T, Burotto M, et al. Nivolumab plus cabozantinib versus sunitinib for advanced renal-cell carcinoma. N Engl J Med 2021;384:829-41.

88 Galle PR, Finn RS, Qin S, et al. Patient-reported outcomes (PROs) from the phase III IMbrave150 trial of atezolizumab (atezo) + bevacizumab (bev) vs sorafenib (SOR) as first-line treatment (tx) for patients (PTS) with unresectable hepatocellular carcinoma (HCC). $J$ Clin Oncol 2020;38:476.

89 Bedke J, Plimack E, Stus V. Health-related quality-of-life (HRQoL) analysis from KEYNOTE-426: pembrolizumab (pembro) plus axitinib (axi) vs sunitinib for advanced renal cell carcinoma (RCC). 35th Annual EAU Congress - Virtual (EAU20V) July 19, 2020, 2020. Available: https://resource-centreuroweborg/resource-centre/ EAU20V/212868/Abstract

90 European Society for Medical Oncology. ESMO-Magnitude of clinical benefit scale, 2020. Available: https://www.esmo.org/ guidelines/esmo-mcbs

91 Marin-Acevedo JA, Chirila RM, Dronca RS. Immune checkpoint inhibitor toxicities. Mayo Clin Proc 2019:94:1321-9.

92 Osorio JC, Ni A, Chaft JE, et al. Antibody-mediated thyroid dysfunction during T-cell checkpoint blockade in patients with nonsmall-cell lung cancer. Ann Oncol 2017;28:583-9.

93 Postow MA, Sidlow R, Hellmann MD. Immune-related adverse events associated with immune checkpoint blockade. N Engl J Med 2018:378:158-68

94 Das S, Johnson DB. Immune-related adverse events and anti-tumor efficacy of immune checkpoint inhibitors. J Immunother Cancer 2019;7:306.

95 Oh DY, Cham J, Zhang L, et al. Immune toxicities elicited by CTLA-4 blockade in cancer patients are associated with early diversification of the T-cell repertoire. Cancer Res 2017;77:1322-30.

96 Subudhi SK, Aparicio A, Gao J, et al. Clonal expansion of CD8 T cells in the systemic circulation precedes development of ipilimumab-induced toxicities. Proc Natl Acad Sci U S A 2016;113:11919-24.

97 Tahir SA, Gao J, Miura Y, et al. Autoimmune antibodies correlate with immune checkpoint therapy-induced toxicities. Proc Natl Acad Sci U S A 2019;116:22246-51.

98 Nishijima TF, Shachar SS, Nyrop KA, et al. Safety and tolerability of PD-1/PD-L1 inhibitors compared with chemotherapy in patients with advanced cancer: a meta-analysis. Oncologist 2017;22:470-9.

99 Brahmer JR, Lacchetti C, Schneider BJ, et al. Management of immune-related adverse events in patients treated with immune checkpoint inhibitor therapy: American Society of Clinical Oncology clinical practice guideline. J Clin Oncol 2018;36:1714-68.
100 Topalian SL, Sznol M, McDermott DF, et al. Survival, durable tumor remission, and long-term safety in patients with advanced melanoma receiving nivolumab. J Clin Oncol 2014;32:1020-30.

101 Couey MA, Bell RB, Patel AA, et al. Delayed immune-related events (DIRE) after discontinuation of immunotherapy: diagnostic hazard of autoimmunity at a distance. J Immunother Cancer 2019;7:165.

102 El Osta B, Hu F, Sadek R, et al. A meta-analysis of immune-related adverse events (irAE) of immune checkpoint inhibitors (ICl) from cancer clinical trials. Annals of Oncology 2016;27:vi369.

103 Xing P, Zhang F, Wang G, et al. Incidence rates of immune-related adverse events and their correlation with response in advanced solid tumours treated with NIVO or NIVO+IPI: a systematic review and meta-analysis. J Immunother Cancer 2019;7:341.

104 Ghisoni E, Wicky AM, Latifyan S, et al. Long-Lasting, irreversible and late-onset immune-related adverse events (irAEs) from immune checkpoint inhibitors (ICls): a real-world data analysis. J Clin Oncol 2020;38:e15095.

105 National Comprehensive Cancer Network. National comprehensive cancer network (NCCN) clinical practice guidelines in oncology (NCCN Guidelines $\AA$ ). Management of immunotherapy-related toxicities v1.2021, 2021. Available: https://www.nccn.org/ professionals/physician_gls/pdf/immunotherapy.pdf

106 Puzanov I, Diab A, Abdallah K, et al. Managing toxicities associated with immune checkpoint inhibitors: consensus recommendations from the Society for immunotherapy of cancer (SITC) toxicity management Working group. J Immunother Cancer 2017;5:95.

107 Haanen JBAG, Carbonnel F, Robert C, et al. Management of toxicities from immunotherapy: ESMO clinical practice guidelines for diagnosis, treatment and follow-up. Ann Oncol 2017;28:iv119-42.

108 Martins F, Sykiotis GP, Maillard M, et al. New therapeutic perspectives to manage refractory immune checkpoint-related toxicities. Lancet Oncol 2019;20:e54-64.

109 Petrelli F, Grizzi G, Ghidini M, et al. Immune-related adverse events and survival in solid tumors treated with immune checkpoint inhibitors: a systematic review and meta-analysis. $J$ Immunother 2020;43:1-7.

110 Ricciuti B, Genova C, De Giglio A, et al. Impact of immune-related adverse events on survival in patients with advanced non-small cell lung cancer treated with nivolumab: long-term outcomes from a multi-institutional analysis. J Cancer Res Clin Oncol 2019;145:479-85.

111 Judd J, Zibelman M, Handorf E, et al. Immune-related adverse events as a biomarker in non-melanoma patients treated with programmed cell death 1 inhibitors. Oncologist 2017;22:1232-7.

112 Suresh K, Psoter KJ, Voong KR, et al. Impact of checkpoint inhibitor pneumonitis on survival in NSCLC patients receiving immune checkpoint immunotherapy. J Thorac Oncol 2019;14:494-502.

113 Dall'Olio FG, Rizzo A, Mollica V, et al. Immortal time bias in the association between toxicity and response for immune checkpoint inhibitors: a meta-analysis. Immunotherapy 2021;13:257-70.

$114 \mathrm{Mi}$ X, Hammill BG, Curtis LH, et al. Use of the landmark method to address immortal person-time bias in comparative effectiveness research: a simulation study. Stat Med 2016:35:4824-36.

115 Schadendorf D, Wolchok JD, Hodi FS, et al. Efficacy and safety outcomes in patients with advanced melanoma who discontinued treatment with nivolumab and ipilimumab because of adverse events: a pooled analysis of randomized phase II and III trials. J Clin Oncol 2017;35:3807-14

116 Santini FC, Rizvi H, Plodkowski AJ, et al. Safety and efficacy of retreating with immunotherapy after immune-related adverse events in patients with NSCLC. Cancer Immunol Res 2018:6:1093-9.

117 Apolo AB, Nadal R, Girardi DM, et al. Phase I study of cabozantinib and nivolumab alone or with ipilimumab for advanced or metastatic urothelial carcinoma and other genitourinary tumors. J Clin Oncol 2020;38:3672-84

118 Lampert EJ, Zimmer A, Padget M, et al. Combination of PARP inhibitor olaparib, and PD-L1 inhibitor durvalumab, in recurrent ovarian cancer: a proof-of-concept phase II study. Clin Cancer Res 2020;26:4268-79.

119 Lipson EJ, Long GV, Tawbi H, et al. CA224-047: a randomized, double-blind, phase II/III study of relatlimab (anti-LAG-3) in combination with nivolumab (anti-PD-1) versus nivolumab alone in previously untreated metastatic or unresectable melanoma. Ann Oncol 2018;29:viii464-5.

120 Ngiow SF, von Scheidt B, Akiba H, et al. Anti-TIM3 antibody promotes T cell IFN- $\gamma$-mediated antitumor immunity and suppresses established tumors. Cancer Res 2011;71:3540-51.

121 Rodriguez-Abreu D, Johnson ML, Hussein MA, et al. Primary analysis of a randomized, double-blind, phase II study of the antiTIGIT antibody tiragolumab (tira) plus atezolizumab (atezo) versus 
placebo plus atezo as first-line (1L) treatment in patients with PDL1-selected NSCLC (CITYSCAPE). J Clin Oncol 2020;38:9503.

122 US Food and Drug Administration. Clinical trial endpoints for the approval of cancer drugs and biologics: guidance for industry, 2018. Available: https://www.fda.gov/media/71195/download

123 Xie X, Li X, Yao W. A narrative review: depth of response as a predictor of the long-term outcomes for solid tumors. Trans/ Cancer Res 2021;10:1119-30.

124 Faury S, Foucaud J. Health-related quality of life in cancer patients treated with immune checkpoint inhibitors: a systematic review on reporting of methods in randomized controlled trials. PLOS One 2020;15:e0227344.

125 Sharma P, Hu-Lieskovan S, Wargo JA, et al. Primary, adaptive, and acquired resistance to cancer immunotherapy. Cell 2017:168:707-23

126 Kluger HM, Tawbi HA, Ascierto ML, et al. Defining tumor resistance to PD-1 pathway blockade: recommendations from the first meeting of the SITC immunotherapy resistance Taskforce. J Immunother Cancer 2020;8:e000398.

127 Shankaran V, Ikeda H, Bruce AT, et al. IFNgamma and lymphocytes prevent primary tumour development and shape tumour immunogenicity. Nature 2001;410:1107-11.

128 Spranger S, Bao R, Gajewski TF. Melanoma-intrinsic $\beta$-catenin signalling prevents anti-tumour immunity. Nature 2015;523:231-5

129 Benci JL, Xu B, Qiu Y, et al. Tumor interferon signaling regulates a multigenic resistance program to immune checkpoint blockade. Cell 2016;167:1540-54.

130 Kuang D-M, Zhao Q, Peng C, et al. Activated monocytes in peritumoral stroma of hepatocellular carcinoma foster immune privilege and disease progression through PD-L1. J Exp Med 2009;206:1327-37.

131 Simpson TR, Li F, Montalvo-Ortiz W, et al. Fc-dependent depletion of tumor-infiltrating regulatory $T$ cells co-defines the efficacy of antiCTLA-4 therapy against melanoma. J Exp Med 2013;210:1695-710.

132 Meyer C, Cagnon L, Costa-Nunes CM, et al. Frequencies of circulating MDSC correlate with clinical outcome of melanoma patients treated with ipilimumab. Cancer Immunol Immunother 2014;63:247-57.

133 Keilholz U, Ascierto PA, Dummer R, et al. ESMO consensus conference recommendations on the management of metastatic melanoma: under the auspices of the ESMO guidelines committee. Ann Oncol 2020:31:1435-48.

134 Shan $\mathrm{Q}$, Wang $\mathrm{H}$, Han $\mathrm{X}$, et al. Duration of immunotherapy in patients with advanced lung adenocarcinoma with negative driver genes: case report and literature review. Thorac Cancer 2020;11:3001-6.

135 Lin RS, Lin J, Roychoudhury S, et al. Alternative analysis methods for time to event endpoints under nonproportional hazards: a comparative analysis. Stat Biopharm Res 2020;12:187-98.

136 Othus M, Barlogie B, Leblanc ML, et al. Cure models as a useful statistical tool for analyzing survival. Clin Cancer Res 2012;18:3731-6.

137 Albiges L, Tannir N, Burotto M, et al. 711P nivolumab + ipilimumab $(\mathrm{N}+\mathrm{l})$ vs sunitinib $(\mathrm{S})$ for first-line treatment of advanced renal cell carcinoma (aRCC) in CheckMate 214: 4-year follow-up and subgroup analysis of patients (pts) without nephrectomy. Ann Oncol 2020;31:S559-60.

138 Plimack ER, Rini BI, Stus V, et al. Pembrolizumab plus axitinib versus sunitinib as first-line therapy for advanced renal cell carcinoma (RCC): updated analysis of KEYNOTE-426. J Clin Oncol 2020;38:5001.

139 Gray JE, Villegas A, Daniel D, et al. Three-year overall survival with durvalumab after chemoradiotherapy in stage III NSCLC-update from PACIFIC. J Thorac Oncol 2020;15:288-93.

140 Motzer RJ, Rini BI, McDermott DF, et al. Nivolumab plus ipilimumab versus sunitinib in first-line treatment for advanced renal cell carcinoma: extended follow-up of efficacy and safety results from a randomised, controlled, phase 3 trial. Lancet Oncol 2019:20:1370-85

141 Lenz H-J, Lonardi S, Zagonel V, et al. Nivolumab (NIVO) + lowdose ipilimumab (IPI) as first-line (1L) therapy in microsatellite instability-high/mismatch repair-deficient (MSI-H/dMMR) metastatic colorectal cancer (mCRC): two-year clinical update. J Clin Oncol 2020;38:4040.

142 Motzer RJ, Penkov K, Haanen J, et al. Avelumab plus axitinib versus sunitinib for advanced renal-cell carcinoma. N Engl J Med 2019;380:1103-15.

143 Gutzmer R, Stroyakovskiy D, Gogas H, et al. Atezolizumab, vemurafenib, and cobimetinib as first-line treatment for unresectable advanced BRAFV600 mutation-positive melanoma (IMspire150): primary analysis of the randomised, double-blind, placebo-controlled, phase 3 trial. Lancet 2020;395:1835-44.

144 de Coaña YP, Wolodarski M, Poschke I, et al. Ipilimumab treatment decreases monocytic MDSCs and increases CD8 effector memory T cells in long-term survivors with advanced melanoma. Oncotarget 2017:8:21539-53.

145 Goldman JW, Crino L, Vokes EE, et al. Nivolumab (nivo) in patients (pts) with advanced (adv) NSCLC and central nervous system (CNS) metastases (Mets). J Clin Oncol 2016;34:9038.

146 Long GV, Atkinson V, Ascierto PA, et al. Effect of nivolumab on health-related quality of life in patients with treatment-naïve advanced melanoma: results from the phase III CheckMate 066 study. Ann Oncol 2016;27:1940-6.

147 Cella D, Escudier B, Saggi SS, et al. 714P time to deterioration in quality of life in previously untreated patients with advanced renal cell carcinoma (aRCC) in CheckMate 214. Ann Oncol 2020;31:S562.

148 Garassino MC, Gadgeel S, Esteban E, et al. Patient-reported outcomes following pembrolizumab or placebo plus pemetrexed and platinum in patients with previously untreated, metastatic, non-squamous non-small-cell lung cancer (KEYNOTE-189): a multicentre, double-blind, randomised, placebo-controlled, phase 3 trial. Lancet Oncol 2020;21:387-97.

149 Reck M, Ciuleanu T-E, Dols MC, et al. Nivolumab (NIVO) + ipilimumab (IPI) + 2 cycles of platinum-doublet chemotherapy (chemo) vs 4 cycles chemo as first-line (1L) treatment (tx) for stage IV/recurrent non-small cell lung cancer (NSCLC): CheckMate 9LA. $J$ Clin Oncol 2020;38:9501.

150 Finn RS, Qin S, Ikeda M, et al. IMbrave150: updated overall survival (OS) data from a global, randomized, open-label phase III study of atezolizumab (atezo) + bevacizumab (bev) versus sorafenib (sor) in patients (pts) with unresectable hepatocellular carcinoma (HCC). $J$ Clin Oncol 2021;39:267.

151 Yau T, Kang Y-K, Kim T-Y, et al. Efficacy and safety of nivolumab plus ipilimumab in patients with advanced hepatocellular carcinoma previously treated with sorafenib: the CheckMate 040 randomized clinical trial. JAMA Oncol 2020;6:e204564.

152 Brahmer JR, Ciuleanu T-E, Schenker M, et al. 67P survival of responders to nivolumab (NIVO) + ipilimumab (IPI) as first-line treatment for advanced NSCLC in CheckMate 227, part 1. Ann Oncol 2020;31:S1444-5.

153 Borghaei H, Pluzanski A, Caro RB. Nivolumab plus ipilimumab as first-line treatment for patients with advanced non-small cell lung cancer with brain metastases: results from CheckMate 227. Cancer Res 2020;80.

154 Emamekhoo H, Olsen M, Carthon BC, et al. Safety and efficacy of nivolumab plus ipilimumab (NIVO+IPI) in patients with advanced renal cell carcinoma (aRCC) with brain metastases: interim analysis of CheckMate 920. J Clin Oncol 2019;37:4517.

155 Herbst RS, Garon EB, Kim D-W, et al. Five year survival update from KEYNOTE-010: pembrolizumab versus docetaxel for previously treated, programmed Death-Ligand 1-positive advanced NSCLC. J Thorac Oncol 2021;16:1718-32.

156 Rittmeyer A, Barlesi F, Waterkamp D, et al. Atezolizumab versus docetaxel in patients with previously treated non-small-cell lung cancer (oak): a phase 3, open-label, multicentre randomised controlled trial. Lancet 2017;389:255-65.

157 Sezer A, Kilickap S, Gümüș M, et al. Cemiplimab monotherapy for first-line treatment of advanced non-small-cell lung cancer with PD-L1 of at least 50\%: a multicentre, open-label, global, phase 3 , randomised, controlled trial. Lancet 2021;397:592-604.

158 Paz-Ares L, Luft A, Vicente D, et al. Pembrolizumab plus chemotherapy for squamous non-small-cell lung cancer. $N$ Engl $J$ Med 2018;379:2040-51.

159 West H, McCleod M, Hussein M, et al. Atezolizumab in combination with carboplatin plus nab-paclitaxel chemotherapy compared with chemotherapy alone as first-line treatment for metastatic non-squamous non-small-cell lung cancer (IMpower130): a multicentre, randomised, open-label, phase 3 trial. Lancet Oncol 2019;20:924-37.

160 Paz-Ares L, Ciuleanu T-E, Cobo M, et al. First-Line nivolumab plus ipilimumab combined with two cycles of chemotherapy in patients with non-small-cell lung cancer (CheckMate 9LA): an international, randomised, open-label, phase 3 trial. Lancet Oncol $2021 ; 22: 198-211$

161 Socinski MA, Jotte RM, Cappuzzo F, et al. Atezolizumab for firstline treatment of metastatic nonsquamous NSCLC. N Engl $J$ Med 2018;378:2288-301. 